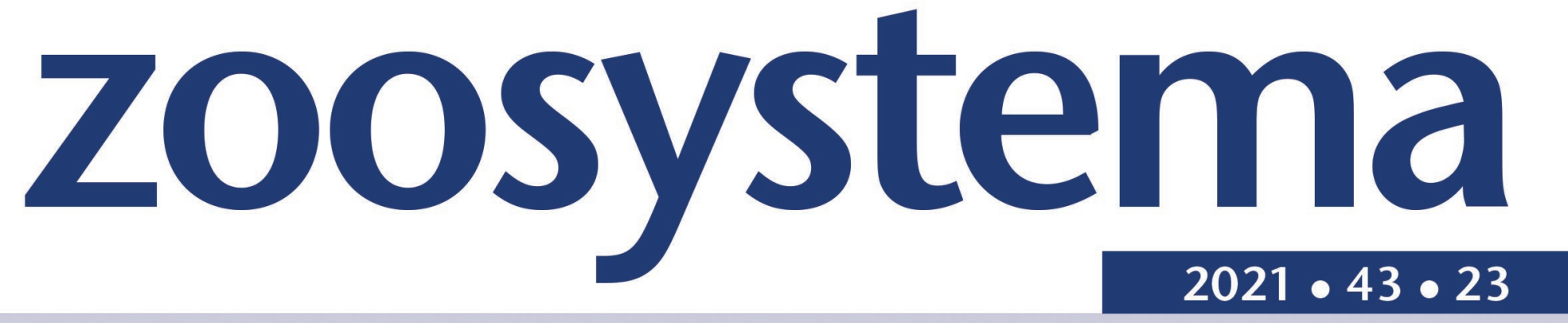

New records of Dryinidae Haliday, 1833 (Hymenoptera, Chrysidoidea) from Iran

Hossein Ali DERAFSHAN, Massimo OLMI, Mostafa GHAFOURI MOGHADDAM, Elham NADER \& Ehsan RAKHSHANI 
Directeur de LA publication / Publication diRECTOR: Bruno David

Président du Muséum national d'Histoire naturelle

RÉDACTRICE EN CHEF / EDITOR-IN-CHIEF: Laure Desutter-Grandcolas

ASSISTANTE DE RÉDACTION / AsSISTANT EDITOR: Anne Mabille (zoosyst@mnhn.fr)

Mise en PAge / PAgE LAYOUt: Anne Mabille

COMITÉ SCIENTIFIQUE / SCIENTIFIC BOARD:

Nesrine Akkari (Naturhistorisches Museum, Vienne, Autriche)

Maria Marta Cigliano (Museo de La Plata, La Plata, Argentine)

Serge Gofas (Universidad de Málaga, Málaga, Espagne)

Sylvain Hugel (CNRS, Université de Strasbourg, France)

Marco Isaia (Università degli Studi di Torino, Turin, Italie)

Rafael Marquez (CSIC, Madrid, Espagne)

Jose Christopher E. Mendoza (Lee Kong Chian Natural History Museum, Singapour)

Annemarie Ohler (MNHN, Paris, France)

Jean-Yves Rasplus (INRA, Montferrier-sur-Lez, France)

Wanda M. Weiner (Polish Academy of Sciences, Cracovie, Pologne)

COUVERTURE / COVER:

Anteon pubicorne (Dalman, 1818), female, habitus, lateral view.

Zoosystema est indexé dans / Zoosystema is indexed in:

- Science Citation Index Expanded (SciSearch ${ }^{\circledR}$ )

- ISI Alerting Services ${ }^{\circledR}$

- Current Contents ${ }^{\circledR} /$ Agriculture, Biology, and Environmental Sciences ${ }^{\circledR}$

- Scopus ${ }^{\circledR}$

Zoosystema est distribué en version électronique par / Zoosystema is distributed electronically by:

- BioOne ${ }^{\circledR}$ (http://www.bioone.org)

Les articles ainsi que les nouveautés nomenclaturales publiés dans Zoosystema sont référencés par / Articles and nomenclatural novelties published in Zoosystema are referenced by:

- ZooBank ${ }^{\circledR}$ (http://zoobank.org)

Zoosystema est une revue en flux continu publiée par les Publications scientifiques du Muséum, Paris / Zoosystema is a fast track journal published by the Museum Science Press, Paris

Les Publications scientifiques du Muséum publient aussi / The Museum Science Press also publish:

Adansonia, Geodiversitas, Anthropozoologica, European Journal of Taxonomy, Naturae, Cryptogamie sous-sections Algologie, Bryologie, Mycologie.

Diffusion - Publications scientifiques Muséum national d'Histoire naturelle

CP $41-57$ rue Cuvier F-75231 Paris cedex 05 (France)

Tél.: 33 (0)1 40794805 / Fax: 33 (0)1 40793840

diff.pub@mnhn.fr / https://sciencepress.mnhn.fr

(C) Publications scientifiques du Muséum national d'Histoire naturelle, Paris, 2021

ISSN (imprimé / print) : 1280-9551/ ISSN (électronique / electronic): 1638-9387 


\section{New records of Dryinidae Haliday, 1833 (Hymenoptera, Chrysidoidea) from Iran}

Hossein Ali DERAFSHAN

Department of Plant Protection, College of Agriculture, University of Zabol,

P.O. Box 98615-538, I.R. (Iran)

Massimo OLMI
Tropical Entomology Research Center, Via De Gasperi 10, I-01100 Viterbo (Italy)
olmimassimo@gmail.com
Mostafa GHAFOURI MOGHADDAM
Elham NADER
Ehsan RAKHSHANI
Department of Plant Protection, College of Agriculture, University of Zabol,
P.O. Box 98615-538, I.R. (Iran)
rakhshani@uoz.ac.ir;
ghafourim@uoz.ac.ir

Submitted on 21 April 2020 | Accepted on 2 February 2021 | Published on 15 September 2021

urn:Isid:zoobank.org:pub:5469C71F-2050-4422-8CF9-B624BEB55C78

Derafshan H. A., Olmi M., Ghafouri Moghaddam M., Nader E. \& Rakhshani E. 2021. - New records of Dryinidae Haliday, 1833 (Hymenoptera, Chrysidoidea) from Iran. Zoosystema 43 (23): 563-583. https://doi.org/10.5252/zoosystema2021v43a23. http://zoosystema.com/43/23

KEY WORDS

Anteoninae,

Aphelopinae,

Bocchinae,

Dryininae,

Auchenorrhyncha,

Palaearctic,

new records.

MOTS CLÉS

Anteoninae,

Aphelopinae,

Bocchinae,

Dryininae,

Auchenorrhyncha,

Paléarctique,

signalisations nouvelles.

\section{ABSTRACT}

New records of Dryinidae Haliday, 1833 (Hymenoptera, Chrysidoidea) from Iran are listed. Two subfamilies (Anteoninae R. Perkins, 1912 and Aphelopinae R. Perkins, 1912), three genera (Anteon Jurine, 1807; Aphelopus Dalman, 1823; and Bocchus Ashmead, 1893) and five species viz. Anteon abdulnouri Olmi, 1987, An. pubicorne (Dalman, 1818), Aphelopus melaleucus (Dalman, 1818), Ap. orphanidesi Olmi, 1994 and Bocchus hyalinus Olmi, 1998 are newly recorded from Iran. The putative male of Dryinus tamaricicola Rakhshani \& Olmi, 2016 is discovered in Iran and described. Aphelopus orphanidesi is recorded for the first time from Germany and Sweden. The diagnostic characters of the newly known species are re-evaluated. A key to the Iranian Dryinidae (excluding Gonatopodinae Kieffer, 1906) and a brief description is presented and a distribution map is provided for each species.

\section{RÉSUMÉ}

Nouvelles signalisations de Dryinidae Haliday, 1833 (Hymenoptera, Chrysidoidea) d'Iran.

De nouvelles signalisations de Dryinidae Haliday, 1833 (Hymenoptera, Chrysidoidea) d'Iran sont répertoriées. Deux sous-familles (Anteoninae R. Perkins, 1912 et Aphelopinae R. Perkins, 1912), trois genres (Anteon Jurine, 1807, Aphelopus Dalman, 1823 et Bocchus Ashmead, 1893) et cinq espèces, à savoir Anteon abdulnouri Olmi, 1987, An. pubicorne (Dalman, 1818), Aphelopus melaleucus (Dalman, 1818), Ap. orphanidesi Olmi, 1994 et Bocchus hyalinus Olmi, 1998, sont nouvellement signalés en Iran. Le mâle jusqu’ici supposé de Dryinus tamaricicola Rakhshani \& Olmi, 2016 est découvert en Iran et décrit. Aphelopus orphanidesi est signalé pour la première fois en Allemagne et en Suède. Les caractères de diagnose des espèces nouvellement connues sont réévalués. Nous présentons ici une brève description et une clé des Dryinidae iraniens (à l'exclusion des Gonatopodinae Kieffer, 1906) ainsi qu'une carte de répartition pour chaque espèce. 


\section{INTRODUCTION}

The Dryinidae Haliday, 1833 (Hymenoptera, Chrysidoidea) are a small cosmopolitan family known to parasitize Hemiptera Auchenorrhyncha Dumeril, 1806 (Guglielmino \& Olmi 2007; Guglielmino et al. 2013). About 1886 species are attributed to this family (Olmi 2020). All species of Dryinidae are ectophagous, except for the completely endophagous genus Crovettia Olmi, 1984 (not present in Iran) and the first endophagous larval instar of the genus Aphelopus Dalman, 1823 (Olmi \& van Harten 2000). Dryinidae are easily distinguished from other members of Chrysidoidea by ten antennomeres and the chelate female protarsi except for the subfamilies Aphelopinae R. Perkins, 1912 (Aphelopus Dalman, 1823 and Crovettia Olmi, 1984) and Erwiniinae Olmi \& Guglielmino, 2010 (Erwinius Olmi \& Guglielmino, 2010), whose females are achelate (Olmi \& Virla 2014), and capture the hosts by their mandibles and forelegs (Olmi 1994). Males of this interesting family are little known and sexual dimorphism is so strong that opposite sexes cannot be associated without rearing or DNA analysis (Olmi 1984; Olmi et al. 2000). This study could reveal interesting biogeographic patterns and clarify the status of the genera and species. Anyway, the group is sufficiently distinct to warrant recognition at the generic level (Olmi et al. 2019).

The family Dryinidae has remained insufficiently known in Iran, due to the lack of sampling. The few known details about the Dryinidae of Iran refer to studies and descriptions of new species by Derafshan et al. $(2016,2017,2020)$ in the eastern part of the country. Our investigations in the central, NorthEastern and South-Eastern parts of Iran, during 2015-2018, resulted in the first country records of five species representing three genera and two subfamilies records and increased the number of known Dryinidae from 12 to 17 species.

\section{MATERIAL AND METHODS}

Sampling and collection of specimens were done in various habitats (Fig. 1A-F) of the eastern and central provinces of Iran during 2015-2018, using Malaise traps (Fig. 1A, B, D, F), with $75 \%$ ethanol solution as a preservative, sweeping (Fig. 1C), and beating vegetation to knock insects onto sheets. More than seventy dryinid specimens were separated from other collected Hymenoptera and stored in plastic vials in 75\% ethanol and later prepared in Alcohol-Xylene-Amyl acetate (AXA protocol: van Achterberg 2009), mounted on cards, labeled, and examined under a Nikon ${ }^{\circ}$ SMZ645 stereomicroscope. Photographs of the external morphological characters of the specimens were taken using a Canon ${ }^{\circledR}$ EOS 700D (Canon ${ }^{\oplus}$ Inc., Japan). Image stacking was performed with ZereneStacker ${ }^{\mathrm{rM}}$ version 1.04. Plates were composed in Photoshop CS6.

Identifications of subfamilies and genera were performed using keys published by Olmi (2006), Olmi \& Virla (2014) and Olmi $\&$ Xu (2015). Females of Dryinidae were identified to the species level by using keys from Olmi \& Xu (2015). Terminology of morphological characters followed that of Olmi (1984), Olmi \& Xu (2015), Kawada et al. (2015) and Azevedo et al. (2018). The term "metapectal-propodeal complex" is here used in the sense of Kawada et al. (2015). It corresponds to the term "propodeum" sensu Olmi (1984), Olmi \& Virla (2014) and Olmi \& Xu (2015). The terms "disc of metapectal-propodeal complex" and "first abdominal tergum" sensu Kawada et al. (2015), used here, correspond to the terms "dorsal surface of propodeum" and "posterior surface of propodeum", sensu Olmi (1984), Olmi \& Virla (2014) and Olmi \& Xu (2015). The names of cells and veins of the fore wing are here used in the sense of Azevedo et al. (2018). The correspondence between old and new names is the following (the first name is the old name): median cell: radial cell $(\mathrm{R})$; submedian cell: first cubital cell $(1 \mathrm{Cu})$; marginal cell: second radial 1 cell (2R1); first brachial cell: second cubital cell $(2 \mathrm{Cu})$; stigmal vein: second radial cross\&radial sector (2r-rs\&Rs); metacarp: poststigmal abscissa of radial 1 (PostabR1). In the text, cells and veins will be named by their respective abbreviations, including costal cell (C).

Geographic coordinates, when not available on specimen labels, were obtained using Google Earth ${ }^{\circ}$. Maps were prepared using SimpleMappr (Shorthouse 2010).

\section{ABBREVIATIONS \\ Biogeographic regions \\ AFR Afrotropical; \\ E-PAL Eastern-Palaearctic; \\ W-PAL Western-Palaearctic.}

\section{Depositories and institutions}

AMNH American Museum of Natural History, New York;

CNC Canadian National Collection of Insects, Ottawa;

DPPZ Department of Plant Protection, College of Agriculture, University of Zabol;

HNHM Hungarian Natural History Museum, Budapest;

HTC Hubert Tussac's collection, Cahors, France (now in the Museum of Natural History, Genève, Switzerland);

MNHN Muséum national d'Histoire naturelle, Paris;

MOLC Massimo Olmi's Collection, c/o DAFNE Department, University of Tuscia, Viterbo;

MRSN Museo Regionale di Scienze Naturali di Torino;

MTC Michael von Tschirnhaus collection, c/o Senckenberg Deutsches Entomologisches Institut (DEI), Müncheberg;

NHMUK The Natural History Museum, London;

NHRS Naturhistoriska Riksmuseet, Stockholm;

NUM NTNU University Museum, Trondheim;

USNM National Museum of Natural History, Washington, District of Columbia;

ZIL Zoological Institute, Lund;

ZMUH Zoological Museum of the University, Helsinki.

The collected specimens from Iran are retained in the Department of Plant Protection, College of Agriculture, University of Zabol, Muséum national d'Histoire naturelle, Paris and the Massimo Olmi's Collection, c/o DAFNE Department, University of Tuscia.

\section{RESULTS}

Two subfamilies (Anteoninae R. Perkins, 1912 and Aphelopinae) and three genera of Dryinidae are recorded for the first time from Iran represented by five species Anteon abdulnouri Olmi, 1987, Anteon pubicorne (Dalman, 1818), Aphelopus 

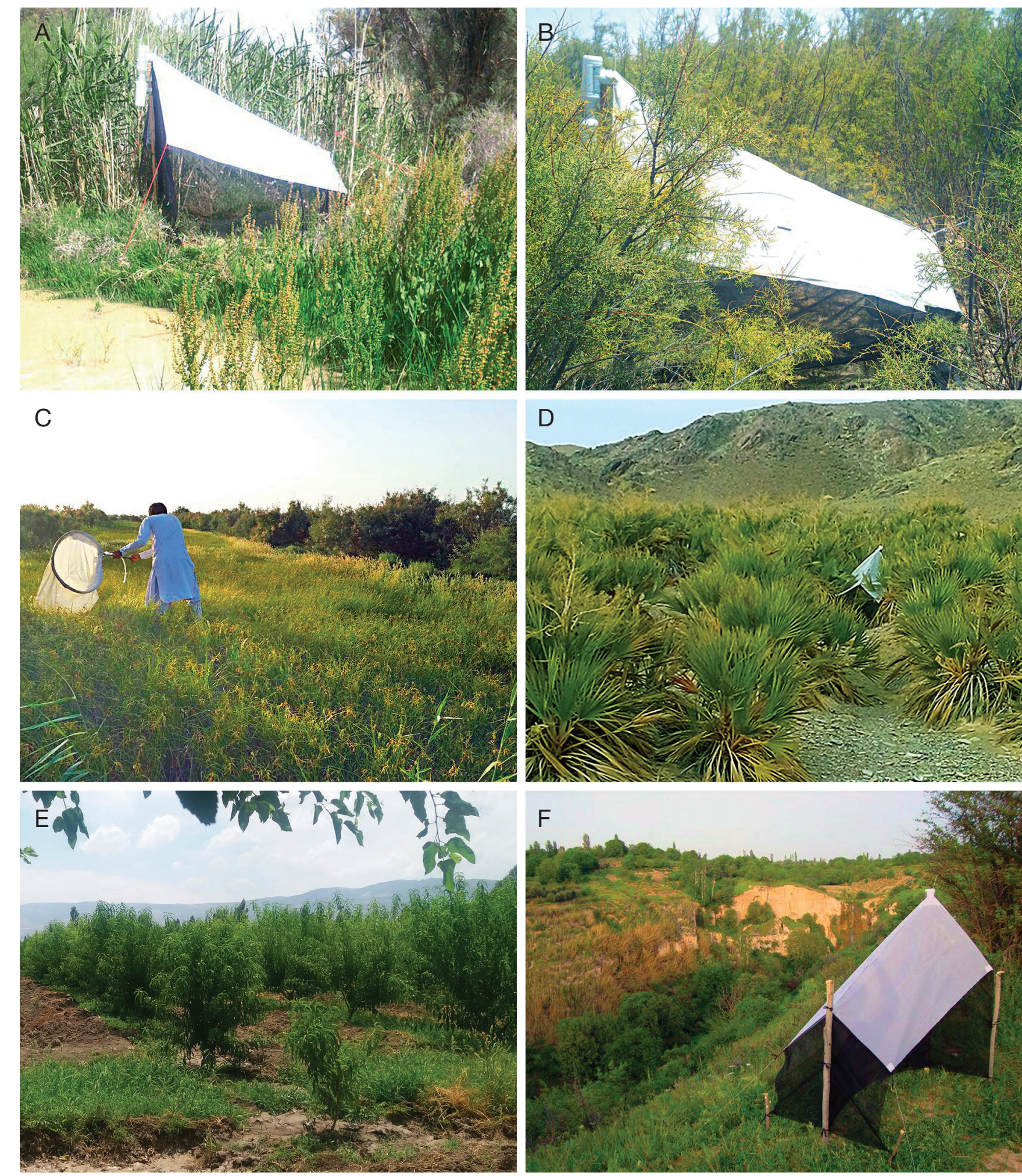

FIG. 1. - The representative habitats, where most of the dryinid specimens were collected: A-C, Sistan area; $\mathbf{A}$, mixed vegetations near the river; $\mathbf{B}$, among the salt cedars around Hamoon lake; C, undergrowth covered with nut grass; D, Baluchestan area, malaise trap among Mazari palms; E, and F, North-Eastern part of Iran, open Fruit orchards.

melaleucus (Dalman, 1818), Aphelopus orphanidesi Olmi, 1994, and Bocchus hyalinus Olmi, 1998. In addition, the putative male of Dryinus tamaricicola Rakhshani \& Olmi, 2016 in Derafshan et al. 2016 was collected for the first time. Three previously recorded species (Mirodryinus atlanticus Olmi, 1984; Dryinus gharaeii Olmi, 2005; D. tarraconensis Marshall, 1868) are also listed in this paper. The newly recorded taxa are marked with an asterisk $\left(^{*}\right)$. 


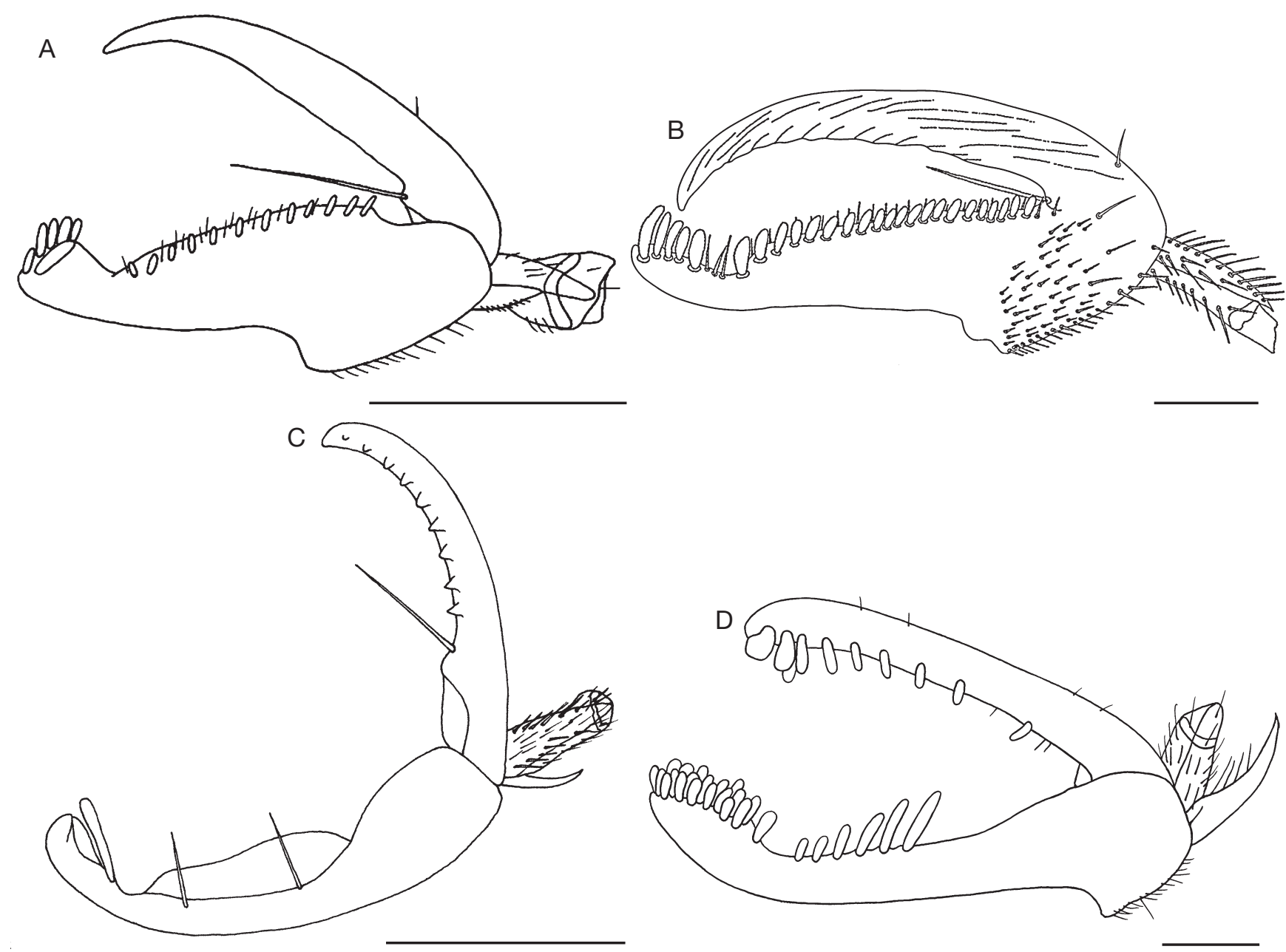

FIG. 2. - Chelae, female: A, Anteon abdulnouri Olmi, 1987 (Lebanon - Redrawn from Olmi 1987); B, Anteon pubicorne (Dalman, 1818) (Italy - redrawn from Olmi, 1984); C, Bocchus hyalinus Olmi, 1998 (Oman - Redrawn from Olmi 1998); D, Dryinus tamaricicola Rakhshani \& Olmi, 2016 (Iran - redrawn from Derafshan et al. 2016). Scale bars: $100 \mu \mathrm{m}$.

\section{TAXONOMIC ACCOUNTS}

Class INSECTA Linnaeus, 1758

Order HYMENOPTERA Linnaeus, 1758

Family DRYINIDAE Haliday, 1833

Subfamily AnTEOninae R. Perkins, 1912*

Genus Anteon Jurine, 1807*

Anteon Jurine, 1807: 302.

TYPE SPECIES. — Anteon jurineanum Latreille, 1809, by subsequent monotypy.

Anteon abdulnouri Olmi, 1987*

(Figs 2A; 3A; 4; 5)

Anteon abdulnouri Olmi, 1987: 33.

TYPE LOCALITY. — Lebanon, Fanar (holotype o , AMNH).

MATERIAL EXAMINED. — Iran (new record) $\bullet 10^{\top}$; MOLC; Sistan-o Baluchestan province, Zabol; 23.V.2016; H. A. Derafshan leg.; swept on weeds; Nim-215 • 1 ơ; MNHN; same locality label; 23.IV.2015; swept on mix vegetations; Nim-45 • 1 \%; MNHN; North Khorasan province, Qale Khan; 05.VI.2018; Z. Rahmani leg.; swept in orchard undergrowth.

Yemen • 1 ơ; MOLC; Lahj; VI-VII.1998; A. van Harten \& A. Sallam leg.; Malaise trap • 2 ơ, 9 \%; MOLC; Lahj; VIII.1998; A. van Harten \& A. Sallam leg.

Distribution In IRAN (Fig. 13A). — North Khorasan and Sistan-o Baluchestan Provinces.

GENERAL Distribution (Fig. 13A). — AFR, E-PAL and W-PAL.

\section{DESCRIPTION}

Female (Figs 2A; 4)

Head dull, completely granulate (Fig. 4B); frontal line complete (Fig. 4A). Pronotum dull, almost completely granulate, only with posterior margin unsculptured (Fig. 4C); posterior surface shorter than mesoscutum (5:9); pronotal tubercle reaching tegula (Fig. 4C); mesoscutum shiny, slightly granulate (Fig. 4C); notauli reaching approximately 0.3-0.4 $\times$ length of mesoscutum (Fig. 4C); metapectal-propodeal disc dull, reticulate rugose, with strong transverse posterior keel 

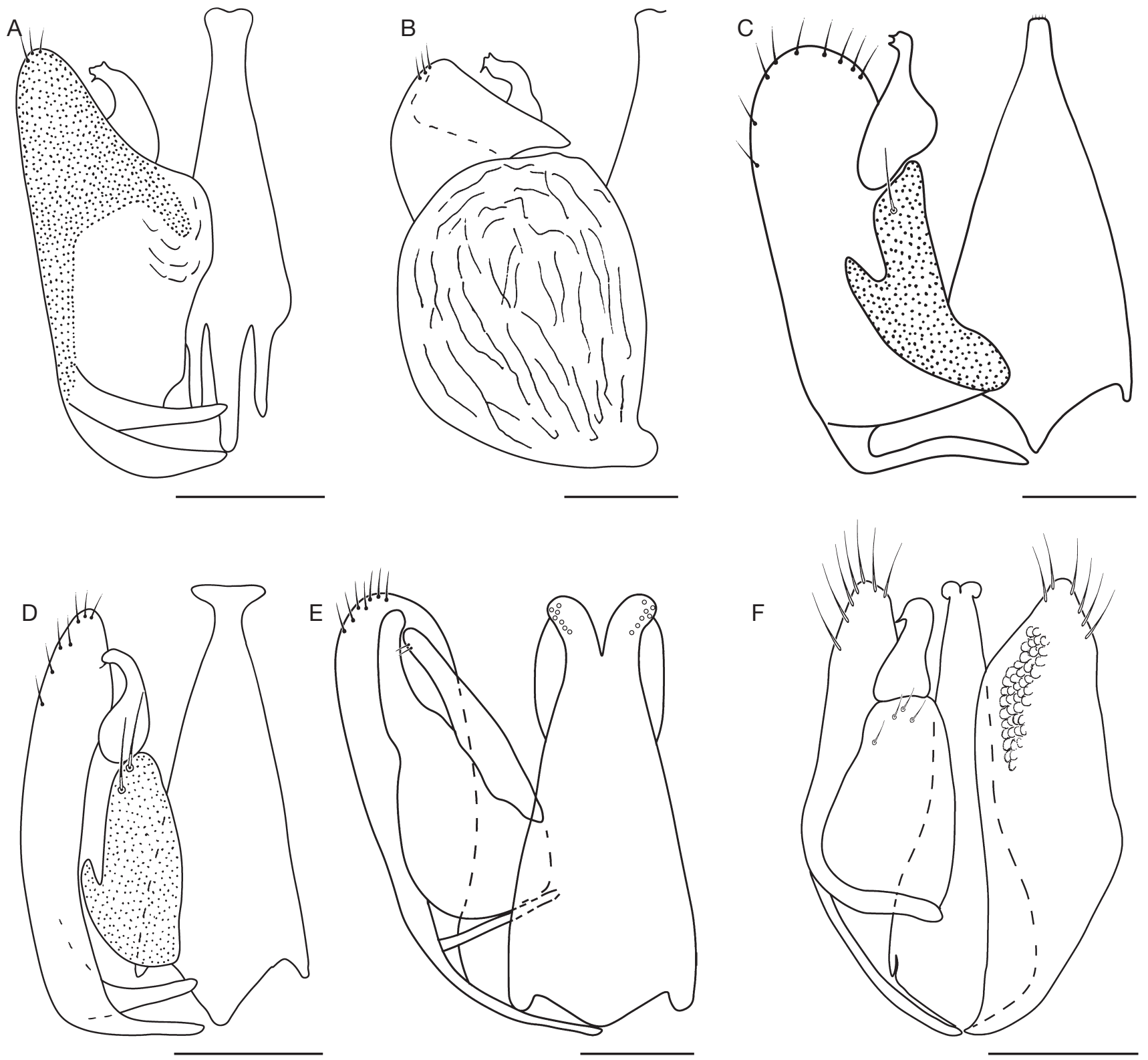

FIG. 3. - Male genitalia: A, Anteon abdulnouri Olmi, 1987 (Yemen - Redrawn from Olmi \& Van Harten 2000); B, Anteon pubicorne (Dalman, 1818) (England redrawn from Olmi, 1984); C, Aphelopus melaleucus (Dalman, 1818) (France - original drawing); D, Aphelopus orphanidesi Olmi, in Olmi \& Orphanides 1994 (Holotype, Cyprus - redrawn from Olmi \& Orphanides 1994); E, Bocchus hyalinus Olmi, 1998 (Oman - redrawn from Olmi et al. 2019); F, Dryinus tamaricicola Rakhshani \& Olmi, in Derafshan et al. 2016 (Iran - original drawing). Scale bars: A-E, 50 m; F, $100 \mu \mathrm{m}$.

(Fig. 4D); propodeal declivity reticulate rugose, without longitudinal keels (Fig. 4D). Protarsomere 5 with two rows of approximately 27 bristles, occasionally with two rows of approximately 12-13 lamellae in addition to some bristles (Fig. 2A); apex with about 4-6 lamellae (Fig. 2A).

\section{Male (Fig. 5)}

Head dull, completely granulate (Fig. 5A, B); frontal line absent (occasionally incomplete) (Fig. 5A); notauli reaching about $0.4 \times$ length of mesoscutum (Fig. $5 \mathrm{C}$ ); metapectalpropodeal complex with strong transverse keel between disc of metapectal-propodeal complex and propodeal declivity (Fig. 5C); disc of metapectal-propodeal complex reticulate rugose (Fig. 5D). Propodeal declivity without longitudinal keels, completely reticulate rugose. Paramere without distal inner pointed process, with inner medial process surrounded by membranous band (Fig. 3A).

\section{REMARKS}

In specimens from Oman, antenna darkened, legs testaceous, except brown metacoxa and clubs of femora; head slightly granulate; mesoscutum dull and strongly granulate. In specimens from Dubai and Afghanistan, frons with unsculptured area in front of anterior ocellus. Frontal line incomplete in some specimens from Afghanistan and Japan, sometimes absent in some Japanese specimens. 



FIG. 4. - Anteon abdulnouri Olmi, 1987, female: A, head, frontal view; B, head, dorsal view; C, mesosoma, dorsal view; D, metapectal-propodeal complex, dorsal view; $\mathbf{E}$, fore wing; $\mathbf{F}$, habitus, lateral view. Scale bars: A-D, $300 \mu \mathrm{m} ; \mathrm{E}, \mathrm{F}, 500 \mu \mathrm{m}$.

Anteon pubicorne (Dalman, 1818)*

(Figs 2B; 3B; 6-8)

Gonatopus pubicornis Dalman, 1818: 87.

TyPe LOCALITY. - Sweden, Småland (holotype , NHRS).
Material EXAMINED. — Iran (new record) • 2 \%; MOLC, MNHN; North Khorasan province, Abshar; 37³2’28”N, 56 56’57”E; 1100 m; 25.IX.2016; B. Motamedinia leg.; Malaise trap.

Distribution In IRAN (Fig. 13A). — North Khorasan province. General Distribution (Fig. 13A). — E-PAL and W-PAL. 

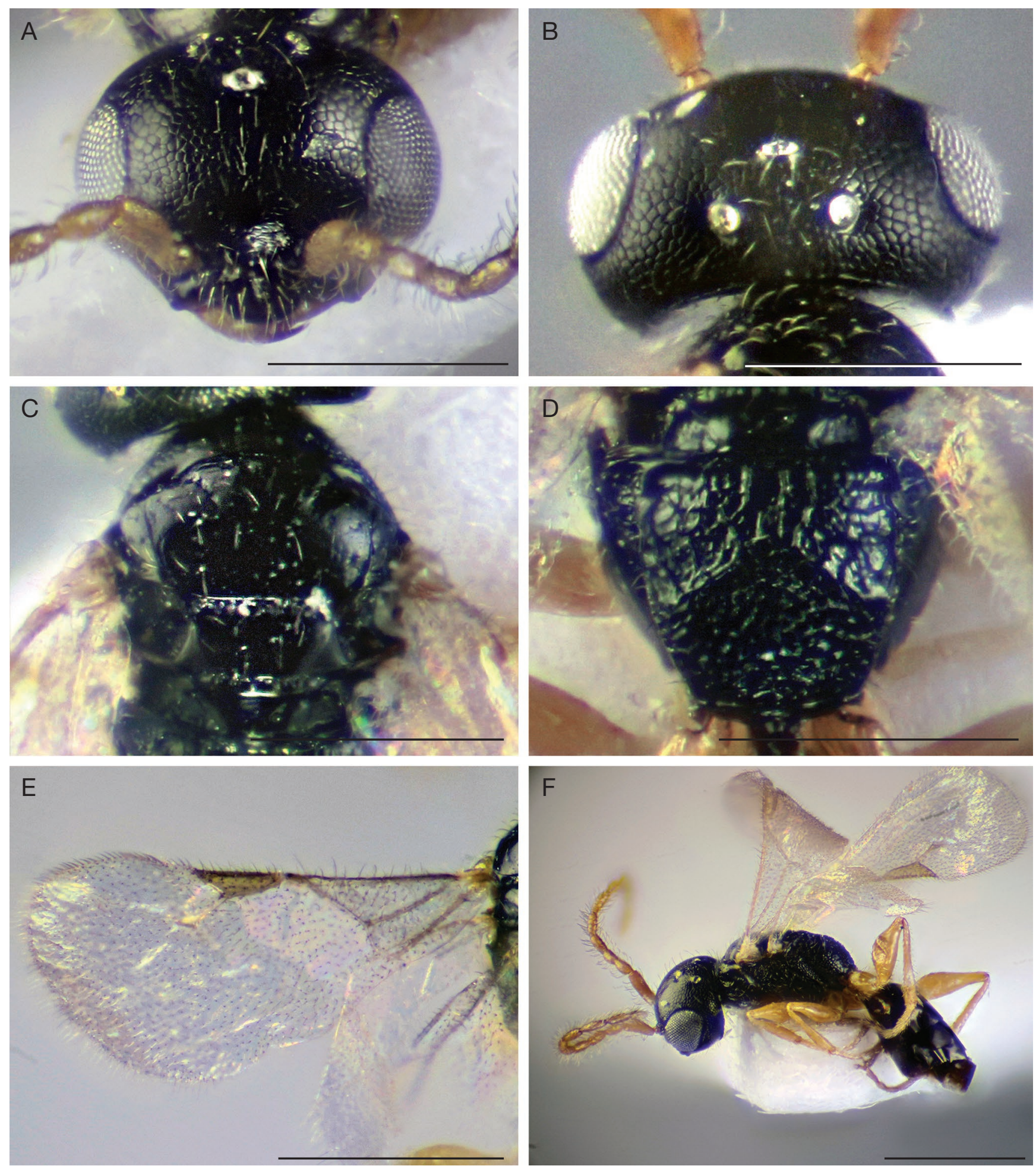

FIG. 5. - Anteon abdulnouri Olmi, 1987, male: A, head, frontal view; B, head, dorsal view; C, mesosoma, dorsal view; D, metapectal-propodeal complex, dorsal view; E, fore wing; F, habitus, lateral view. Scale bars: A-D, $300 \mu \mathrm{m} ; \mathrm{E}, \mathrm{F}, 500 \mu \mathrm{m}$.

\section{DESCRIPTION}

Female (Figs 2B; 6-8)

Head shiny, smooth, finely or strongly punctate, unsculptured among punctures (Fig. 6A-C); frons with two lateral keels around orbits directed towards antennal toruli (Fig. 6B); frontal line incomplete or absent in small specimens (Fig. 6B); temple present (Fig. 6C). Pronotum shiny, strongly punctate (Fig. 6H); pronotal tubercle reaching tegula (Fig. 6H); notauli reaching approximately $0.5-0.6 \times$ length of mesoscutum (Fig. $6 \mathrm{H}$ ); metapectal-propodeal complex reticulate rugose, with strong transverse keel between disc of metapectal-propodeal complex and propodeal declivity (Fig. 6H). Propodeal 

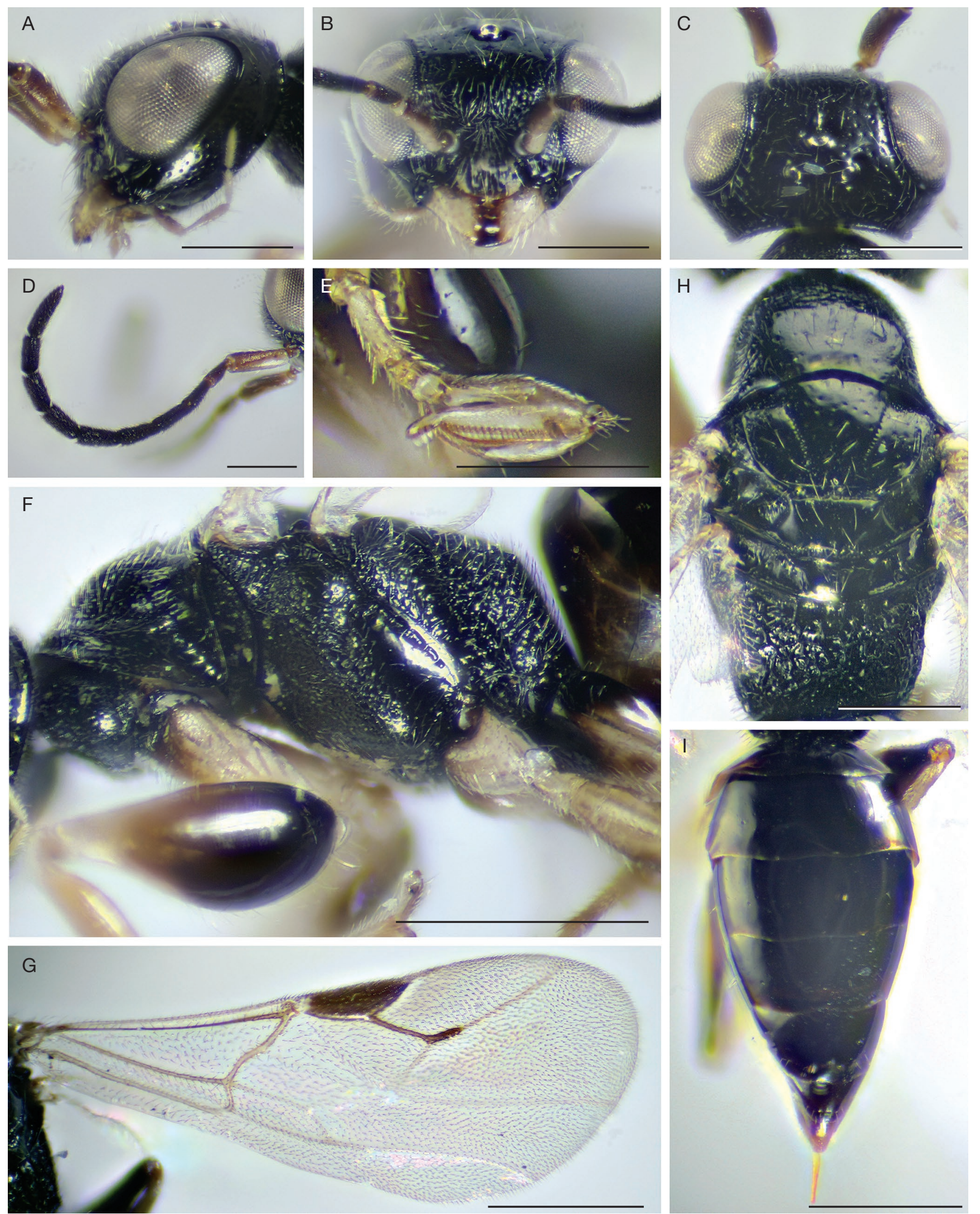

FIG. 6. - Anteon pubicorne (Dalman, 1818), female: A, head, lateral view; B, head, frontal view; C, head, dorsal view; $\mathbf{D}$, antenna, lateral view; E, chela; $\mathbf{F}$, mesosoma, lateral view; G, fore wing; H, metapectal-propodeal complex and mesosoma, dorsal view; I, metasoma, dorsal view. Scale bars: A-E, H, 300 um; F, G, I, 500 Hm. 


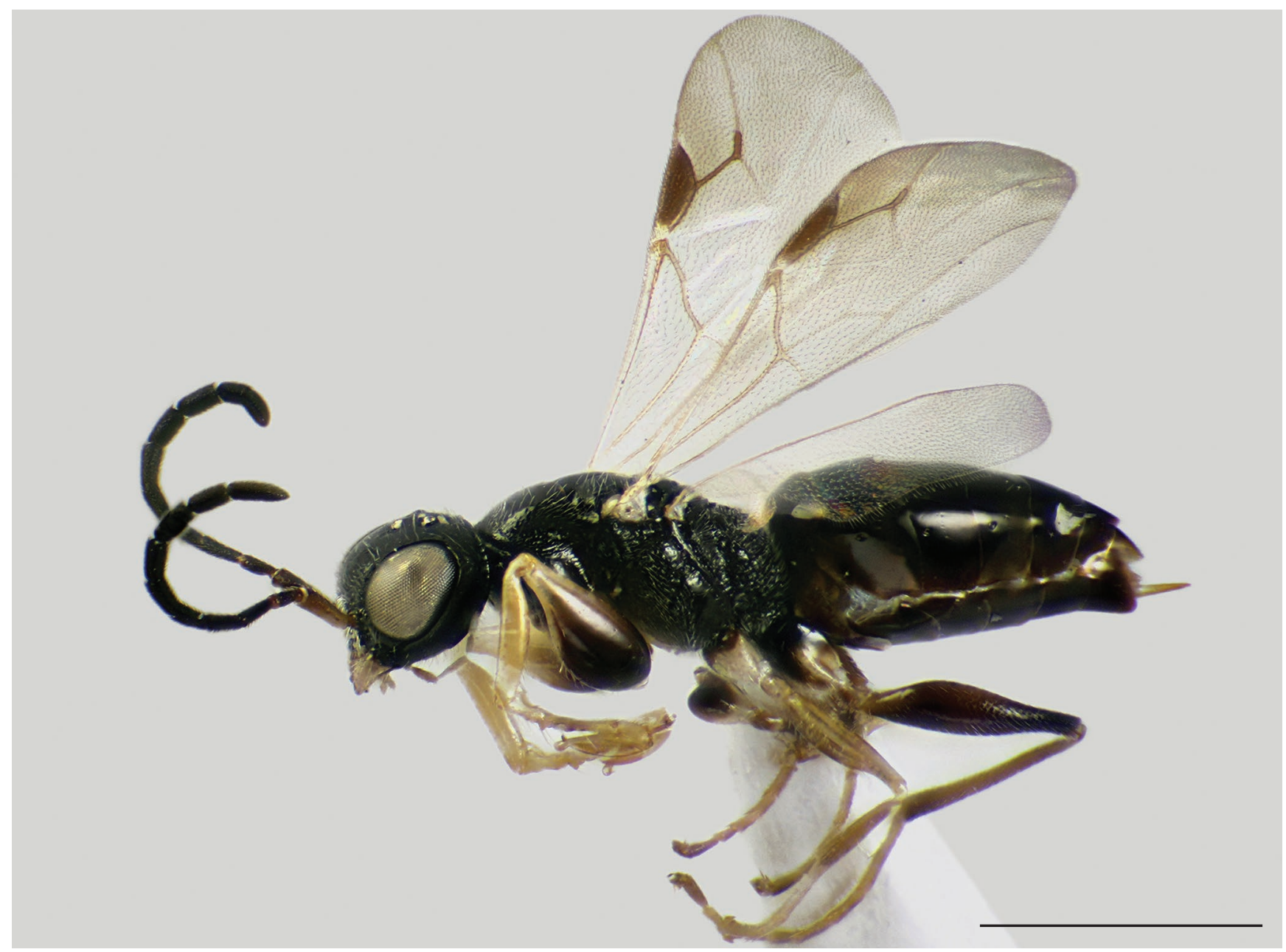

FIG. 7. - Anteon pubicorne (Dalman, 1818), female, habitus, lateral view. Scale bar: $1 \mathrm{~mm}$.

declivity with two longitudinal keels and median area usually as rugose as lateral areas (Fig. 6I); occasionally median area totally or partly shiny and smooth (Fig. 6I). Protarsomere 1 approximately 0.66 as long as protarsomere 4 ; protarsomere 2 produced into hook; protarsomere 5 with one row of 20-23 lamellae; distal apex with about 5-6 lamellae (Figs 2B; 6E).

\section{Male}

Not collected in Iran.

\section{REMARK}

In some specimens from Sweden, head uniformly brown or with cupreous tints; scape or scape + pedicel testaceous; antenna testaceous, except antennomeres 3-6 brown; mesosoma uniformly brown or with cupreous tints; legs occasionally with clubs of femora brown.

Subfamily Aphelopinae R. Perkins, 1912*

$$
\text { Genus Aphelopus Dalman, 1823* }
$$

Aphelopus Dalman, 1823: 8.
TyPe SPECIES. - Dryinus atratus Dalman, 1823; designated by Westwood (1840).

\section{Aphelopus melaleucus (Dalman, 1818)* \\ (Figs 3C; 8)}

Gonatopus melaleucus Dalman, 1818: 82.

TYPE LOCALITY. - Sweden, Västergötland (holotype $\$$, NHRS).

Material EXAMINED. - Iran (new record) $120^{\text {; }}$; MOLC; Isfahan province, Nisian; 32 $59^{\prime} 5.61^{\prime \prime} \mathrm{N}, 52^{\circ} 28^{\prime} 13.91^{\prime \prime} \mathrm{E}, 2191 \mathrm{~m}$; 8-28. VIII.2015; E. Nader leg.; Malaise trap in orchard; code-17 $110^{\text {; }}$; MNHN; same locality label; 7-31.VII.2015; code-7; • 1 ơ; DPPZ; same locality label; 8-21.VII.2015; code-14 - 1 0'; DPPZ; Khorasane Razavi province; 15.IX.2016; B. Motamedinia leg.; Malaise trap.

DisTribution IN IRAN (Fig. 13B). - Isfahan and Khorasan-e Razavi provinces.

General Distribution (Fig. 13B). - E-PAL and W-PAL.

\section{DESCRIPTION}

Male (Figs 3C; 8)

Black; occasionally antennomeres 9-10 light. Head dull, granulate (Fig. 8A, B); frontal line incomplete, not present 

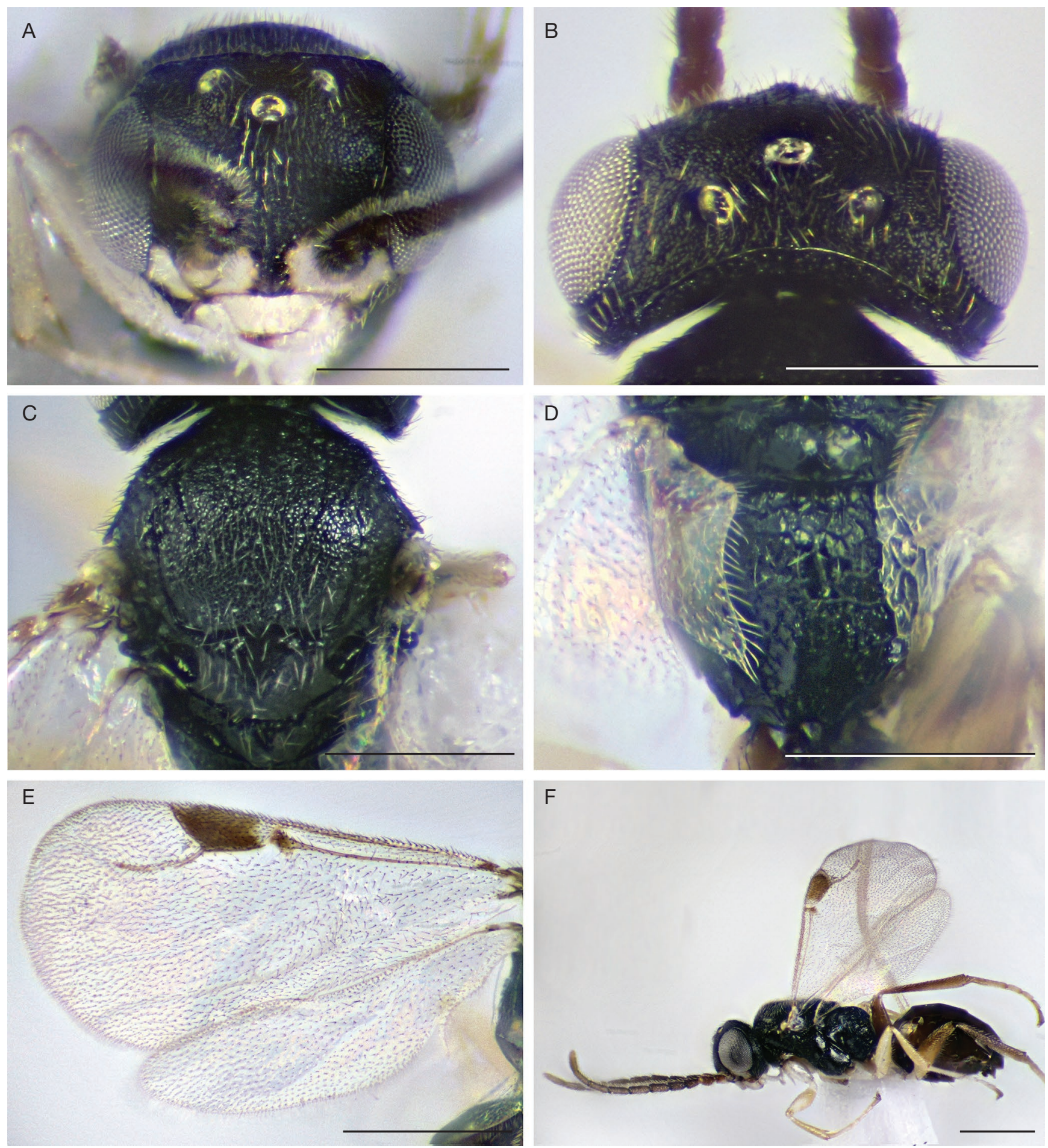

FIG. 8. - Aphelopus melaleucus (Dalman, 1818), male: A, head, frontal view; B, head, dorsal view; C, mesosoma, dorsal view; D, metapectal-propodeal complex, dorsal view; E, fore wing; F, habitus, lateral view. Scale bars: A-D, $300 \mu \mathrm{m} ; \mathrm{E}, \mathrm{F}, 500 \mu \mathrm{m}$.

in front of anterior ocellus, high carina shaped on anterior half of frons (Fig. 8A). Mesoscutum occasionally with median scutal line (Fig. 8C); notauli reaching approximately $0.5 \times$ length of mesoscutum (Fig. 8C); metapectal-propodeal complex dull, reticulate rugose; propodeal declivity with two complete longitudinal keels, median area shiny, almost smooth, with few irregular slight keels, less rugose than lateral areas (Fig. 8D). Distal apex of aedeagus not tridentate, not trumpet-shaped, basivolsella with one subdistal bristle and with outer basal process little or very protruding (Fig. 3C).

Female

Not collected in Iran. 

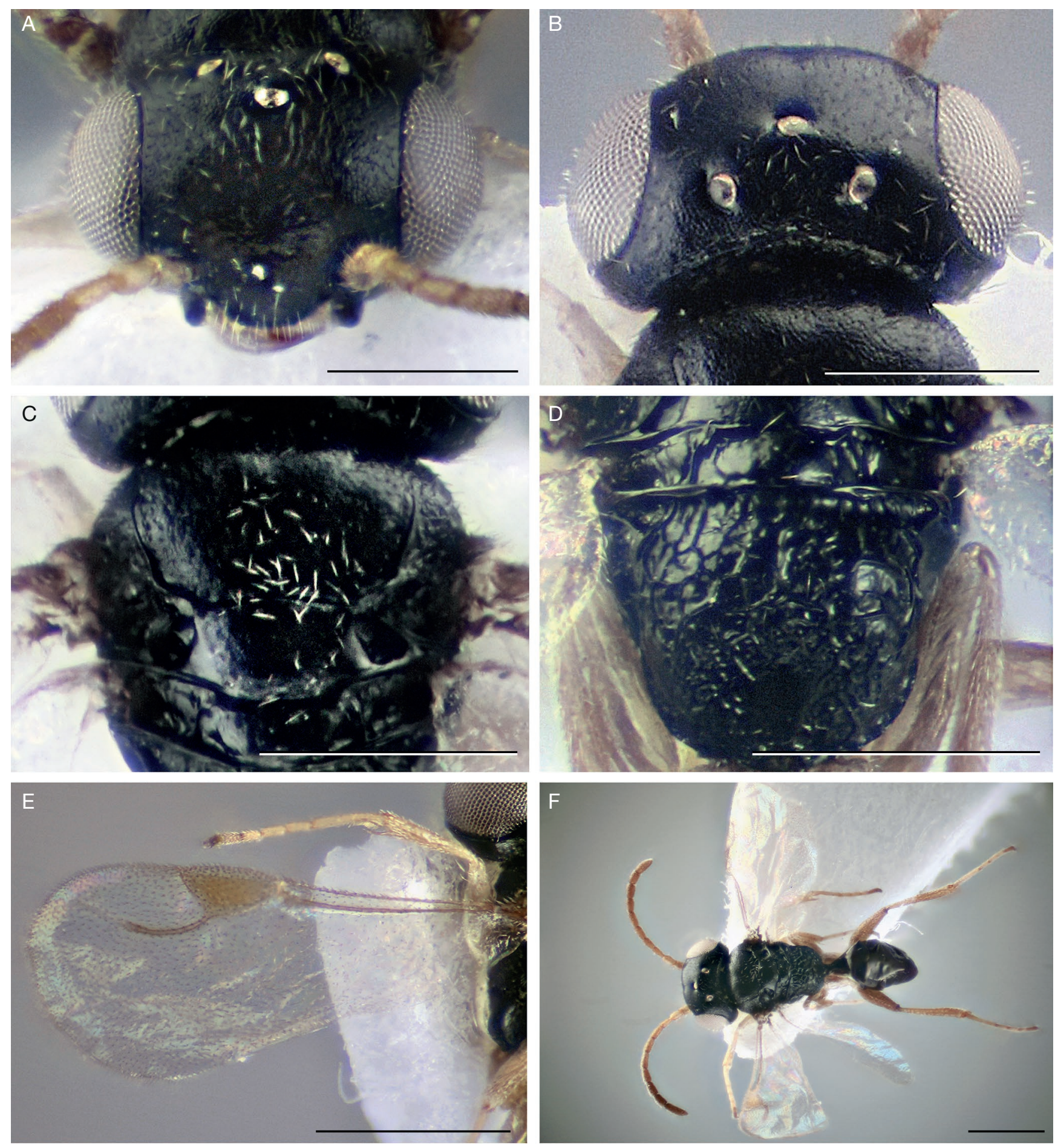

FIG. 9. - Aphelopus orphanidesi Olmi, 1994, female: A, head, frontal view; B, head, dorsal view; C, mesosoma, dorsal view; D, metapectal-propodeal complex, dorsal view; E, fore wing; F, habitus, dorsal view. Scale bars: A-D, $300 \mu \mathrm{m} ; \mathrm{E}, \mathrm{F}, 500 \mu \mathrm{m}$.

\section{REMARKS}

In many male specimens from England (Awbridge), head and body brown-testaceous, except mandible, clypeus and few small spots situated between antennal toruli and on gena whitish or yellowish; other specimens from above mentioned locality testaceous-reddish. In other specimens from all countries where this species is present, head occasionally completely black, without light spots.
Aphelopus orphanidesi Olmi, 1994*

(Figs 3D; 9, 10)

Aphelopus orphanidesi Olmi, in Olmi \& Orphanides, 1994: 408.

TYPE LOCALITY. - Cyprus, Pakhna (holotype $0^{7}$ and paratype $\$$, AMNH).

Material eXAmined. - Holotype. Cyprus • ơ; AMNH; Pakhna II.1993. 

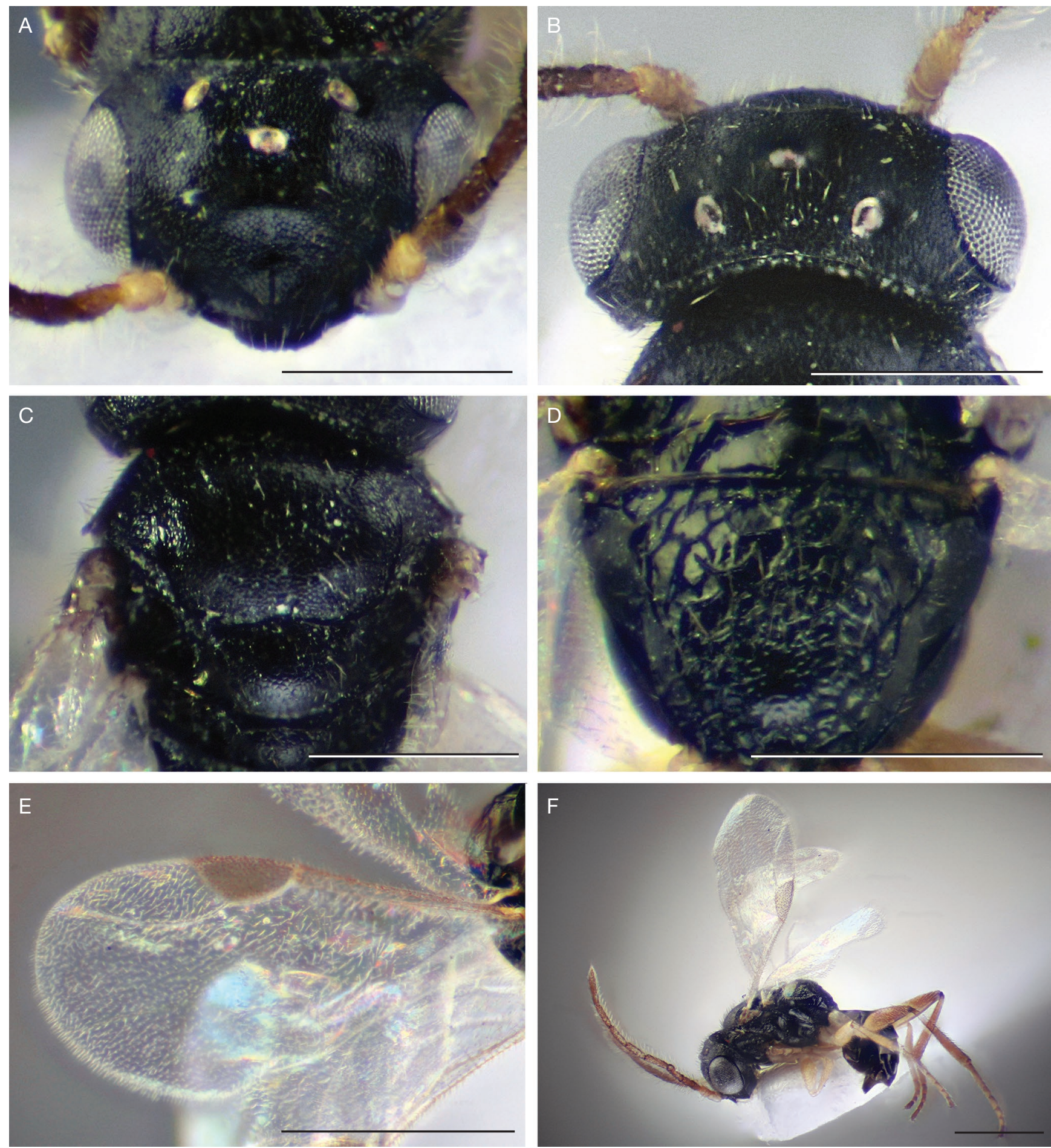

FIG. 10. - Aphelopus orphanidesi Olmi, 1994, male: A, head, frontal view; B, head, dorsal view; C, mesosoma, dorsal view; D, metapectal-propodeal complex, dorsal view; E, fore wing; F, habitus, lateral view. Scale bars: A-D, $300 \mu \mathrm{m} ; \mathrm{E}, \mathrm{F}, 500 \mu \mathrm{m}$.

Paratypes. Cyprus • 7 \%; AMNH; Pakhna; II.1993; G. Orphanides leg.; reared from Zygina rhamni Ferrari $\bullet 60^{\pi} ;$ AMNH $\bullet 1$ ơ; USNM; - 2 ơ, 2\%; MRSN; same date and locality label $\bullet 1$ \%; NHMUK; same date and locality label.

France 1 o; NHMUK; Bouches-du-Rhône, Fonscolombe; 24.VI.1986; M. de V. Graham leg. • 3 ơ; MOLC, HTC; PyrénéesOrientales, Le Perthus; $42^{\circ} 27.04^{\prime} \mathrm{N}, 02^{\circ} 51.82^{\prime} \mathrm{E}$; 1 .VIII.1995; J. P. Sarthou leg.; Malaise trap.
Germany (new record) • 1 \%; MOLC; Schleswig-Holstein, Frörup $S$ of Flensbu Forest; Werner Barkemeyer leg.; L2727; ex Michael von Tschirnhaus collection 1 \% ; MTC; $1.5 \mathrm{~km}$ NNE of Tensfeld; $54^{\circ} 03.37^{\prime} \mathrm{N}, 10^{\circ} 19.33^{\prime} \mathrm{E}$; 2.V.1989; Hans Meyer leg.; K965; ex Michael von Tschirnhaus collection.

Iran (new record) • 10 ơ; 1 \%; MOLC, DPPZ; Isfahan province, Nisian; 32 ${ }^{\circ} 59^{\prime} 5.61^{\prime \prime} \mathrm{N}, 52^{\circ} 28^{\prime} 13.91$ ”E; $2191 \mathrm{~m}$; 8-28.VIII.2015; E. Nader leg.; code-17; Malaise trap in orchards $\bullet 4$ ơ; DPPZ; same 
locality label; 7-31.VII.2015; code-7 • 3 o'; MNHN; same locality label; 8-21.VII.2015; code-14 1 o o $^{\text {; }}$ MOLC; Isfahan province, Najafabad; $32^{\circ} 39^{\prime} \mathrm{N}, 51^{\circ} 23^{\prime} \mathrm{E}$; $1644 \mathrm{~m}$; 7-31.VII.2015; E. Nader leg.; code-9; Malaise trap in orchards.

Italy • 1 o $10^{\text {7 }}$; MOLC; Toscana, Livorno province, Venturina; 8-17.VIII.1998 to 17-31.VIII.1998; 17.VIII-2.IX.1999 to 2-10.

IX.1999; F. Strumia leg.; Malaise trap.

Norway • Frode Ødegaard leg.; NUM.

Sweden (new record) • 1 o'; ZIL; Sk., Dalby; 14-20.VIII.2001;

R. Danielsson leg.; yellow pan trap.

Distribution IN IRAN (Fig. 13B). — Isfahan province.

General distribution (Fig. 13B). - W-PAL.

\section{DESCRIPTION}

Female (Fig. 9A-F)

Black; antenna brown or brown-testaceous, except scape and pedicel yellow (Fig. 9A). Antennomeres in following proportions: 4:3:4:4:5:5:4.5:4:4:6. Head dull, granulate (Fig. 9A, B); frontal line incomplete, only a trace present in anterior third of frons (Fig. 9A). Mesoscutum dull, granulate (Fig. 9C); notauli reaching approximately $0.4 \times$ length of mesoscutum (Fig. 9C); metapectal-propodeal complex with disc reticulate rugose (Fig. 9D); propodeal declivity with wide median area shiny, almost completely unsculptured, surrounded by rugose areolae (Fig. 9D). 2r-rs\&Rs vein regularly curved (Fig. 9E).

Male (Figs 3D; 10).

Head dull, granulate (Fig. 10A, B); frontal line incomplete, only present in anterior third of frons (Fig. 10A). Mesoscutum dull, granulate (Fig. 10C); notauli reaching approximately $0.4 \times$ length of mesoscutum (Fig. 10C); mesoscutellum shiny, slightly granulate (Fig. 10C); metanotum shiny, unsculptured; metapectal-propodeal complex dull, with disc reticulate rugose (Fig. 10D). Propodeal declivity almost completely shiny, unsculptured, surrounded by numerous rugose areolae. $2 \mathrm{r}$-rs\&Rs vein regularly curved (Fig. 10E). Distal apex of aedeagus not tridentate; basivolsella with two subdistal bristles and with an outer basal process (Fig. 3D).

\section{REMARKS}

This species is recorded from Germany and Sweden for the first time. In specimen from Sweden, Dalby, a basivolsella has two subdistal bristles, whereas the other basivolsella has only one subdistal bristle.

Subfamily BOCCHINAE Richards, 1939

Genus Bocchus Ashmead, 1893*

Bocchus Ashmead, 1893: 91.

TyPE SPECIES. - Bocchus flavicollis Ashmead, 1893, by original designation.
Bocchus hyalinus Olmi, 1998*

(Figs 2C; 3E; 11)

Bocchus hyalinus Olmi, 1998: 65.

TYPE LOCALITY. - Oman, $4 \mathrm{~km}$ South of Dhagmar (holotype $\%$, CNC).

MATERIAL EXAMINED. - Iran (new record) • 1 \%; MNHN; Kerman province, Faryab; 5.VIII.2016; M. Arab leg.; Malaise trap in Natural Ecosystem - 1 \%; DPPZ; Sistan-o Baluchestan province, Iranshahr, Daman; $27^{\circ} 25^{\prime} \mathrm{N}, 060^{\circ} 54^{\prime} \mathrm{E}$; 879 m; 18.VI.2016; H. Davari leg.; Malaise trap 2 mounted among planted Mazari palm, Nannorrhops ritchiana (Griff.) Aitch. • 3 \%; DPPZ, MOLC; same locality label; $27^{\circ} 24^{\prime} \mathrm{N}, 060^{\circ} 50^{\prime} \mathrm{E}$; $876 \mathrm{~m}$; 16-26.VI.2016; Malaise trap 4.

Oman • 1 o 5 o'; NHMUK, MOLC; Al-Uyaina - Samail; 30.IV.2006; K. Al-Miqbali leg.; ex. Ommatissus lybicus De Bergevin (Tropiduchidae) on date palm.

Distribution in Iran (Fig. 14A). — Kerman and Sistan-o Baluchestan provinces.

General Distribution (Fig. 14A). — AFR and W-PAL.

\section{DESCRIPTION}

Female (Figs 2C; 11)

Body almost totally yellow-whitish, except ocelli slightly darkened; occasionally mesothoracic axilla and sutures behind mesoscutum, mesoscutellum and metanotum darkened (Fig. 11D-F). Antenna short, with antennomeres 5-9 longer than broad (antennomere 5: 6:3; antennomere 9: 4.5:3.5); head dull, completely granulate and reticulate rugose (Fig. 11A-C); frontal line complete (Fig. 11B). Pronotum crossed by strong transverse impression, reticulate rugose (Fig. 11E); pronotal tubercle reaching tegula (Fig. 11E); lateral regions of prothorax continuous with mesopleuron so that the epicnemium is not exposed (Fig. 11D); notauli posteriorly separated, occasionally hardly visible (Fig. 11E); mesoscutellum very slightly granulate, not rugose (Fig. 11E); metanotum short and rugose (Fig. 11F); metapectal-propodeal disc reticulate rugose; propodeal declivity dull and rugose (Fig. 11F). Petiole very long, much shorter than metasoma (6:27) (Fig. 11F). Protarsomere 5 with one preapical lamella, two bristles and an inner band (Fig. 2C).

Male.

Not collected in Iran.

\section{REMARKS}

In specimens from the United Arab Emirates, mesosoma ferruginous and metasoma brown; mesoscutum strongly reticulate rugose, with notauli almost completely invisible; mesoscutellum with anterior half unsculptured and posterior half reticulate rugose. In contrast, in specimen from Kenya, mesosoma ferruginous and metasoma partly brown and partly ferruginous; mesoscutum strongly reticulate rugose, with notauli completely invisible; mesoscutellum granulate.

Genus Mirodryinus Ponomarenko, 1972

Mirodryinus Ponomarenko, 1972: 673.

TYPE SPECIES. - Radiimancus olmii Móczár, 1983, by original designation. 

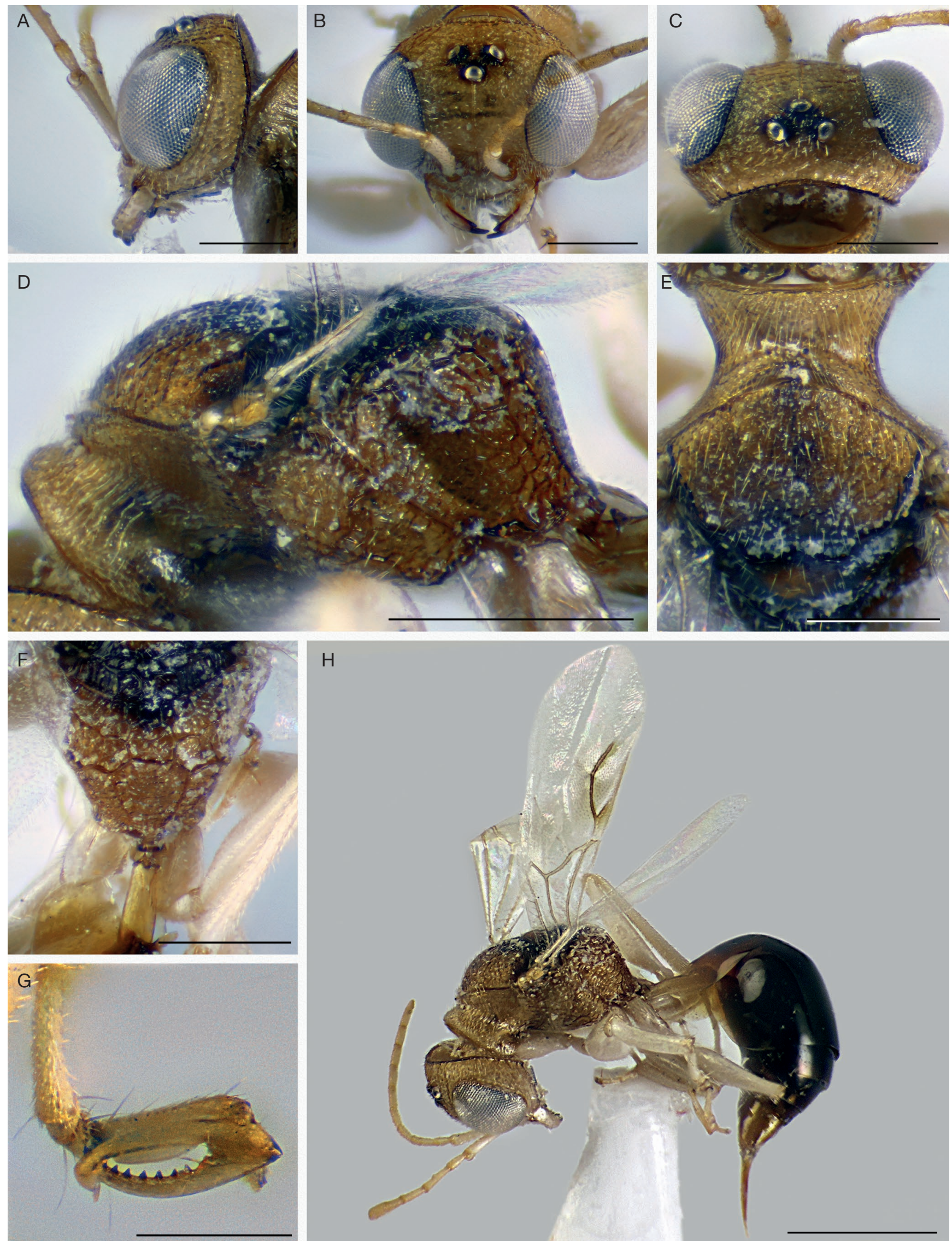

FIG. 11. - Bocchus hyalinus Olmi, 1998, female: A, head, lateral view; B, head, frontal view; C, head, dorsal view; D, mesosoma, lateral view; E, mesosoma,

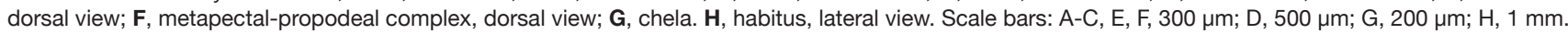



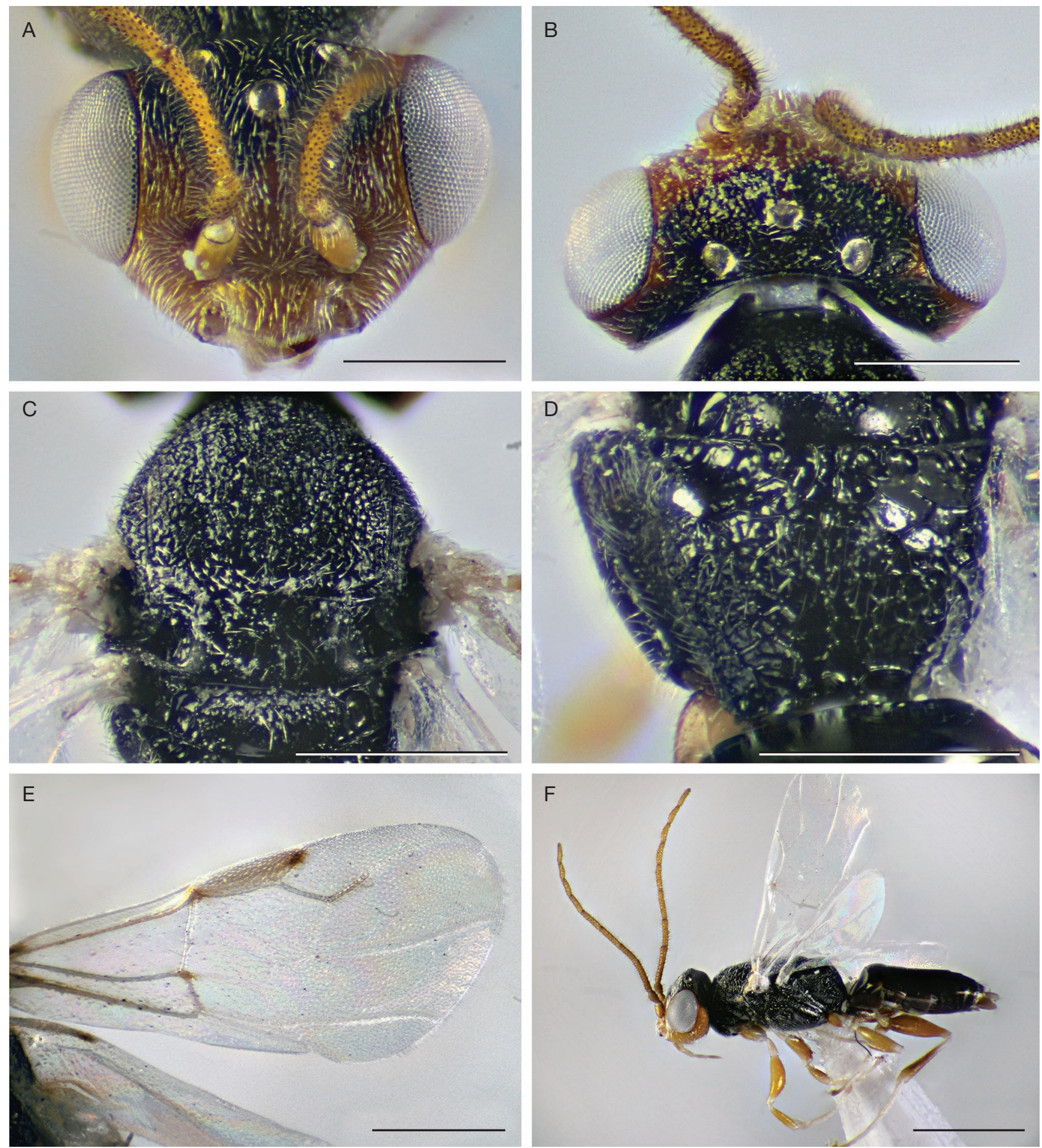

FIG. 12. - Dryinus tamaricicola Rakhshani \& Olmi, 2016, male: A, head, frontal view; B, head, dorsal view; C, mesosoma, dorsal view; D, metapectal-propodeal complex, dorsal view; E, fore wing; F, habitus, lateral view. Scale bars: A, B, $300 \mu \mathrm{m} ; \mathrm{C}-\mathrm{E}, 500 \mu \mathrm{m} ; \mathrm{F}, 1 \mathrm{~mm}$.

\section{Mirodryinus atlanticus Olmi, 1984}

Mirodryinus atlanticus Olmi, 1984: 672.

TyPe LOCALITY. - Spain, Canary Islands, Fuerteventura Island, Gran Tarajal (holotype o, ZMUH).
Distribution In IRAN (Fig. 14A). — Sistan-o Baluchestan province. General Distribution (Fig. 14A). — E-PAL and W-PAL.

DESCRIPTION

Reviewed in Derafshan et al. (2017) for males and females, both known in Iran. 


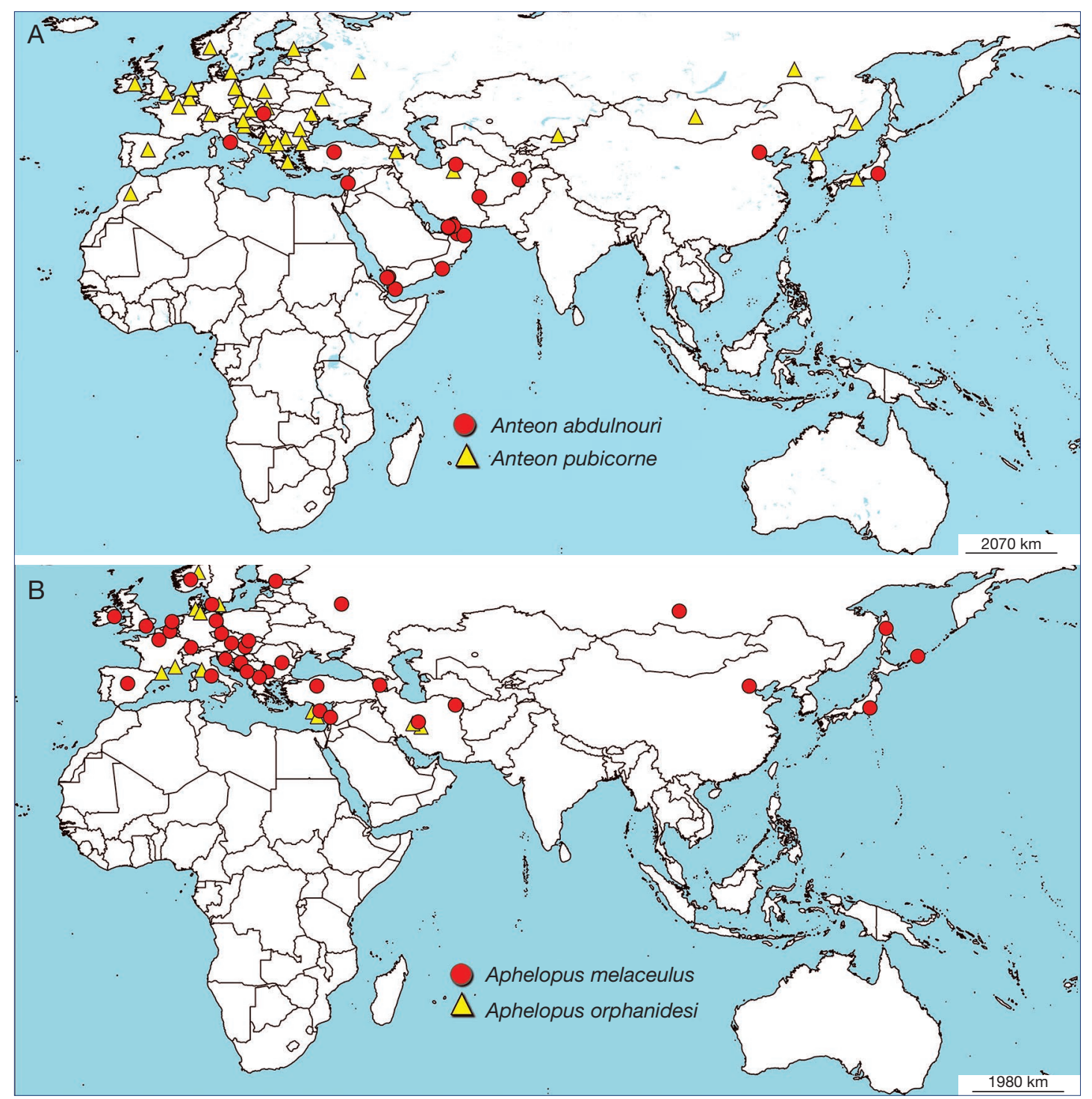

FlG. 13. - Distribution map of the Iranian Anteoninae R. Perkins, 1912 (A): Anteon abdulnouri Olmi, 1987; Anteon pubicorne (Dalman, 1818) and Aphelopinae R. Perkins, 1912 (B): Aphelopus melaleucus (Dalman, 1818); Aphelopus orphanidesi Olmi, in Olmi \& Orphanides 1994 (Hym., Dryinidae) in a worldwide background.

Subfamily DrYININAE Haliday, 1833

Genus Dryinus Latreille, 1804

Dryinus Latreille, 1804: 176.

TYPe SPECIES. - Dryinus collaris (Linnaeus, 1767), by subsequent monotypy (Latreille 1805).

\section{Dryinus gharaeii Olmi, 2005}

Dryinus gharaeii Olmi, 2005: 207.

Type LOCAlity. - Iran, Ilam Province, Chogasabz Region (holotype $\circ$, MOLC).

Distribution IN IRAN (Fig. 14B). — Ilam province.
GENERAL Distribution (Fig. 14B). — W-PAL.

MATERIAL EXAMINED. - Iran • Holotype o; MOLC; Ilam Province, Chogasabz Region, Ilam; 1.IX.2003; Babak Gharaei leg.

\section{DESCRIPTION}

Reviewed in Derafshan et al. (2016).

Male

Unknown.

Dryinus tamaricicola Rakhshani \& Olmi, 2016 (Figs 2D; 3F; 12)

Dryinus tamaricicola Rakhshani \& Olmi, in Derafshan et al. 2016: 412. 


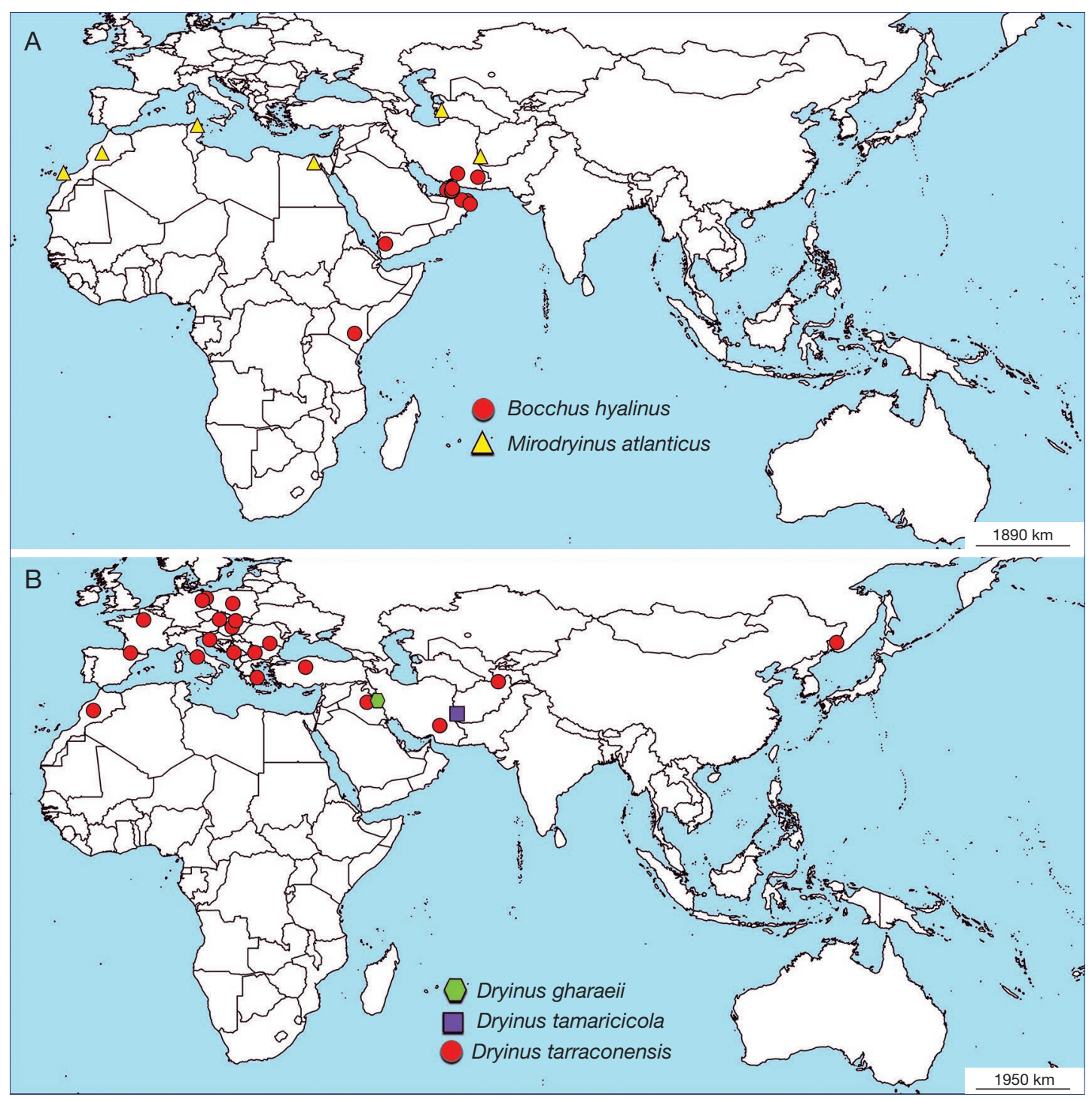

FIG. 14. - Distribution map of the Iranian Bocchinae Richards, 1939 (A): Bocchus hyalinus Olmi, 1998; Mirodryinus atlanticus Olmi, 1984; and Dryininae Haliday, 1833 (B): Dryinus gharaeii Olmi, 2005; Dryinus tamaricicola Rakhshani \& Olmi, 2016; Dryinus tarraconensis Marshall, 1868 (Hym., Dryinidae) in a worldwide background.

TYPE LOCALITY. - Iran, Sistan-o Baluchestan, Zabol (holotype o , MOLC).

MATERIAL EXAMINED. - Iran • 10 ; ; MNHN; Sistan-o Baluchestan province, Zabol; 13.V.2015; H. A. Derafshan leg.; Nim-75; swept on Tamarix stricta 2 o"; DPPZ, MOLC; same locality label; 20.IX.2016; H. A. Derafshan leg.; Nim-243; swept on Tamarix stricta.

DistRIBUTION IN IRAN (Fig. 14B). — Sistan-o Baluchestan province.

General distribution (Fig. 14B). - W-PAL.

\section{DESCRIPTION}

Male (putatively associated with the female - Figs 3F; 12)

Head testaceous, except occiput and part of vertex black; antenna testaceous; mesosoma black; metasoma brown; legs testaceous, except metacoxa partly brown and tarsi whitish. Antennomere 3 about seven times as long as broad (14:2) (Fig. 12A); antennomeres in following proportions: 5:5:14:12:12:10:9:8:7:9; head dull, granulate and partly reticulate rugose (Fig. 12A, B); frontal line absent (Fig. 12A); temple absent (Fig. 12B); greatest breadth of posterior ocelli shorter than OL (3:4) (Fig. 12B); posterior ocelli touching occipital carina (Fig. 12B); occipital carina completely visible on dorsal side of head, where it reaches laterally eyes (Fig. 12B); palpal formula 6/3. Mesoscutum dull, granulate and reticulate rugose (Fig. 12C); notauli reaching about $0.5 \times$ length of mesoscutum (Fig. 12C); mesoscutellum shiny, unsculptured (Fig. 12C); metanotum shiny, unsculptured, except anterior half rugose (Fig. 12C); metapectal-propodeal complex reticulate rugose, without transverse or longitudinal keels (Fig. 12D). Genitalia with distivolsella situated near distal apex of aedeagus (Fig. 3F); paramere with inner side sculptured by many papillae (Fig. 3F). Tibial spurs 1/1/2.

Female

In Derafshan et al. (2016). 


\section{REMARKS}

The male specimens were collected from the same locality as female specimens collected earlier in the same habitat (on Tamarix plants), and by the same methods (with a beating sheet). They were not reared. Thus, here we tentatively assign the male specimens to D. tamaricicola.

\section{Dryinus tarraconensis Marshall, 1868}

Dryinus tarraconensis Marshall, 1868: 204.

TYPE LOCALITY. - Spain, Huesca, near Torla (holotype $\$$, HNHM).

Material eXamined. - Iran • 1 \%; MOLC; Kerman Province, Bam County, Sangemes; 28 56’33.44”N, 5807’52.36”E; 2101 m; 08.III.2015; M. Vafaei leg.; swept on weeds $\bullet 1$ \%; DPPZ; Kerman province, Bam country, Moghoye; 28 $57^{\prime} 24.18^{\prime \prime} \mathrm{N}, 58^{\circ} 06^{\prime} 34.90^{\prime \prime} \mathrm{E}$, 1693 m; 11.VIII.2015; M. Vafaei 1.
Distribution In Iran (Fig. 14B). - Kerman province.

General distribution (Fig. 14B). - E-PAL and W-PAL.

DESCRIPTION

Reviewed in Derafshan et al. (2016).

Male.

Unknown.

\section{Subfamily GonatopodinaE Kieffer in Kieffer \& Marshall 1906}

\section{REMARKS}

This subfamily has been completely reviewed in Derafshan et al. (2020). Distribution in Iran is showed in figure 15.

\section{KEY TO THE IRANIAN DRYINIDAE}

\section{FEMALES}

1. Protarsus chelate (Fig. 2A-D)

— Protarsus not chelate [Aphelopinae R. Perkins, 1912, in Iran only Aphelopus Dalman, 1823] .................... 5

2. Chela with one rudimentary claw (Fig. 2C, D) ............................................................................... 3

- Chela without rudimentary claw (Fig. 2A, B) [Anteoninae R. Perkins, 1912, in Iran only Anteon Jurine, 1807]

3. Median leg with one tibial spur (formula $1 / 1 / 1$ or $1 / 1 / 2$ )

- Median leg without tibial spurs (formula $1 / 0 / 1$ or $1 / 0 / 2$ )

[Gonatopodinae Kieffer, 1906; see Derafshan et al. 2020, for key to Iranian species]

4. Epicnemium concealed (Fig. 11D) [Bocchinae Richards, 1939]

- Epicnemium exposed [Dryininae Haliday, 1833]

5. Head with mandibles, clypeus and lower parts of frontal region white or testaceous

............................................................................................... Aphelopus melaleucus (Dalman, 1818)

- Head with mandibles white or testaceous; clypeus uniformly black; rest of head black (Fig. 9A) Aphelopus orphanidesi Olmi, 1994

6. Protarsomere 4 at most 0.5 as long as protarsomere 1; protarsomere 3 or 4 produced into hook (Fig. 2A) .....

..... Anteon abdulnouri Olmi, 1987

- Protarsomere 4 at least 0.66 as long as protarsomere 1; protarsomere 2 produced into hook (Fig. 2B)..... Anteon pubicorne (Dalman, 1818)

7. Segment 5 of protarsus with inner membranous band, two bristles and with one preapical lamella (Fig. 2C) .

....................................................................................................... Bocchus hyalinus Olmi, 1998

- Segment 5 of protarsus without inner membranous band, with 1 row of about 18 lamellae and 1 row of about 7 bristles, distal apex with approximately 4-10 lamellae Mirodryinus atlanticus Olmi, 1984

8. Enlarged claw with one subapical tooth

Dryinus tarraconensis Marshall, 1868

- Enlarged claw without subapical teeth or with many small subapical teeth

9. Posterior ocelli not touching occipital carina. Enlarged claw with many small subapical teeth

- Posterior ocelli touching occipital carina. Enlarged claw without subapical teeth Dryinus gharaeii Olmi, 2005 Dryinus tamaricicola Rakhshani \& Olmi, 2016 
MALES

1. Fore wing with three cells (Costal, radial and first cubital) enclosed by pigmented veins (Figs 5E, 11E) ...... 2

- Fore wing with only the costal cell enclosed by pigmented veins (Figs 8E, 10E) [Aphelopinae R. Perkins, 1912: in Iran only Aphelopus Dalman, 1823] ............................................................................................... 5

2. Hypopygium not umbrella-shaped [Anteoninae R. Perkins, 1912: in Iran only Anteon Jurine, 1807] ......... 6

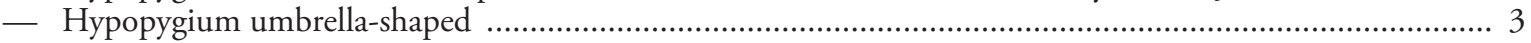

3. Epicnemium concealed [Bocchinae Richards, 1939] .................................................................. 7

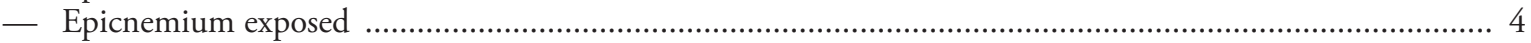

4. Occipital carina complete or incomplete. Dorsal process of paramere absent [Dryininae Haliday, 1833] ..... 8

- Occipital carina absent, occasionally present and complete. Dorsal process of paramere present [Gonatopodinae Kieffer, 1906; see Derafshan et al. 2020 for key to Iranian species]

5. Aedeagus with distal apex not trumpet-shaped (Fig. 3C) ..................... Aphelopus melaleucus (Dalman, 1818)

- Aedeagus with distal apex trumpet-shaped (Fig. 3D) Aphelopus orphanidesi Olmi, 1994

6. Propodeal declivity without longitudinal keels (Fig. 5D). Paramere without inner distal and pointed process (Fig. 3A) .................................................................................................. Anteon abdulnouri Olmi, 1987

- Propodeal declivity with two longitudinal keels. Paramere with distal inner pointed process (Fig. 3B) Anteon pubicorne (Dalman, 1818)

7. Metapectal-propodeal disc with strong transverse posterior keel and two complete longitudinal keels, areolae very wide (Fig. 11F). Genitalia with distivolsella articulated along inner margin of basivolsella (Fig. 3E) ........ Bocchus hyalinus Olmi, 1998

- Metapectal-propodeal disc without longitudinal or transverse keel, areolae very small. Genitalia with distivolsella articulated on distal margin of basivolsella ........................................... Mirodryinus atlanticus Olmi, 1984

8. Antennal segments broad, with segment 3 less than six times as long as broad

Dryinus tarraconensis Marshall, 1868

- Antennal segments slender, with segment 3 seven or more than seven times as long as broad Dryinus tamaricicola Rakhshani \& Olmi, 2016

\section{DISCUSSION}

Few studies have been conducted on the Dryinidae fauna of Iran, with most of the papers restricted to descriptions of species collected sporadically (Olmi 1984, 2005; Olmi \& $\mathrm{Xu}$ 2015). Before the present study, three subfamilies (Bocchinae, Dryininae and Gonatopodinae), five genera (Dryinus, Echthrodelphax R. Perkins, 1903, Gonatopus Ljungh, 1810, Haplogonatopus R. Perkins, 1905 and Mirodryinus) and twelve species were known from Iran (Derafshan et al. 2016, 2017, 2020). With the new records reported herein the totals are increased to five subfamilies (Fig. 16), eight genera and 17 species.

Some species listed in the current study, are known exclusively on the basis of only one sex. Only females are known for An. pubicorne, B. hyalinus, D. gharaeii, and D. tarraconensis and only the male in Ap. melaleucus. Both sexes are known for three species (An. abdulnouri, $M$. atlanticus, and D. tamaricicola). With respect to our collection of D. tamaricicola, because of the pronounced morphological difference between female and male in Dryinus species, the males we collected were putatively associated with the female. We believe the association is reasonable, particularly because of the aforementioned concordance of the sexes in collection location, habitat and method. Nonetheless, molecular data are necessary for confirmation.

From the biogeographical point of view, all the nine species we collected are found in the following sub-regions: An. abdulnouri (E-PAL and W-PAL); An. pubicorne (E-PAL and W-PAL); Ap. melaleucus (E-PAL and W-PAL); $A p$. orphanidesi (W-PAL); B. hyalinus (W-PAL); $M$. atlanticus (E-PAL and W-PAL); D. gharaeii (W-PAL); D. tamaricicola (W-PAL); D. tarraconensis (W-PAL). Two species, D. gharaeii and $D$. tamaricicola, are provisionally considered as endemic, but since Dryinidae have been poorly collected in Iran, their geographic distribution could be larger. Anteon abdulnouri and Bocchus hyalinus have already been recorded from the Afrotropical region (Olmi 1998 ; Olmi \& Xu 2015). We expect that more dryinids are common to both (southern part of) Iran and the Afrotropical region, as provinces located in the south-eastern and southern parts of Iran share similar climatic and floral conditions (Derafshan et al. 2016, 2020; Ghafouri Moghaddam et al. 2019; Heraty et al. 2019). However, further field research is necessary to verify this hypothesis.

To date, there are no host records for Dryinidae collected in Iran. Known hosts of all the species considered in this study belong to the family Cicadellidae Latreille, 1802, except $B$. hyalinus whose host belongs to the family Tropiduchidae 


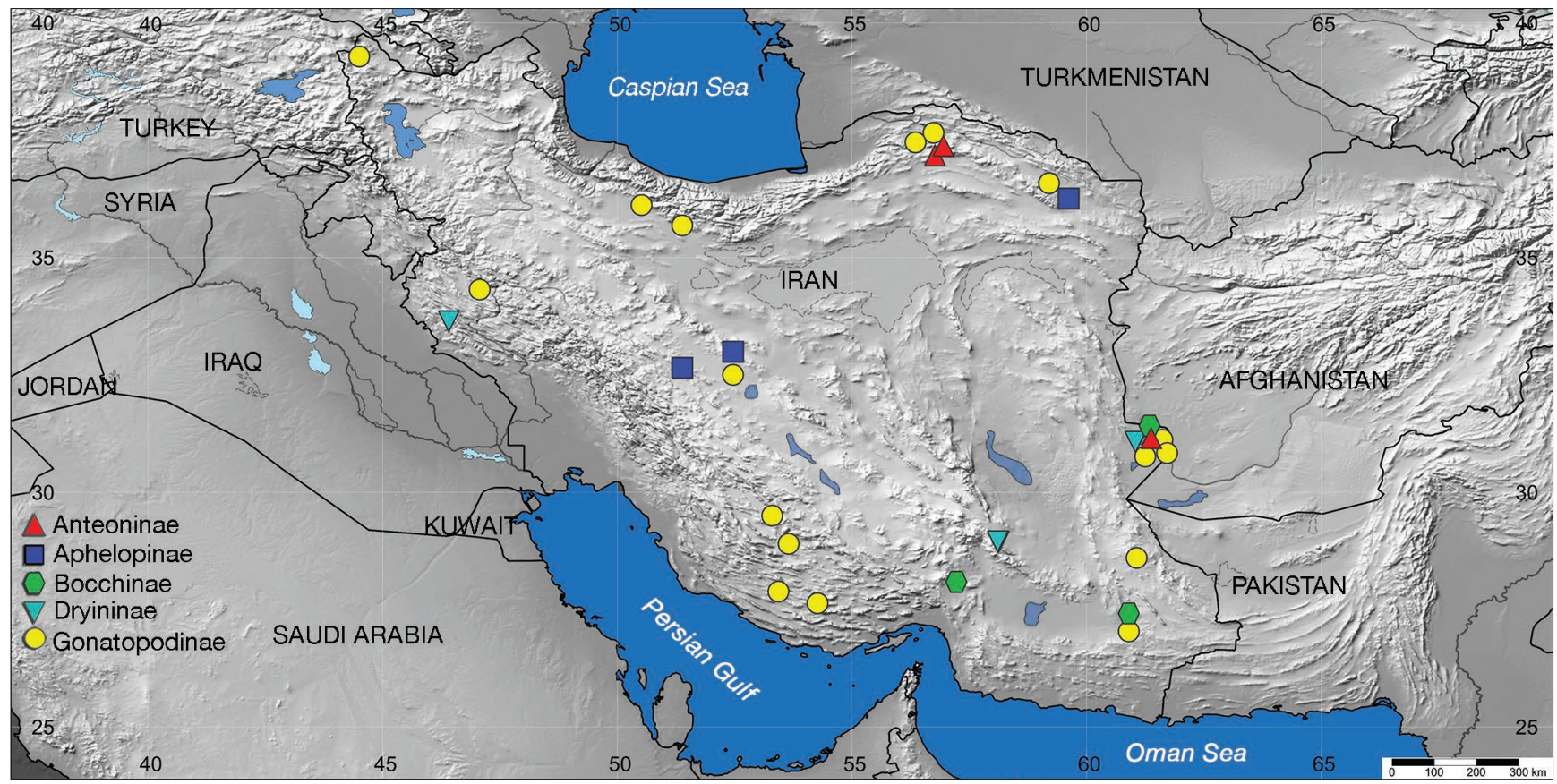

FIG. 15. - Distribution map of all subfamilies of Dryinidae Haliday, 1833 in Iran.

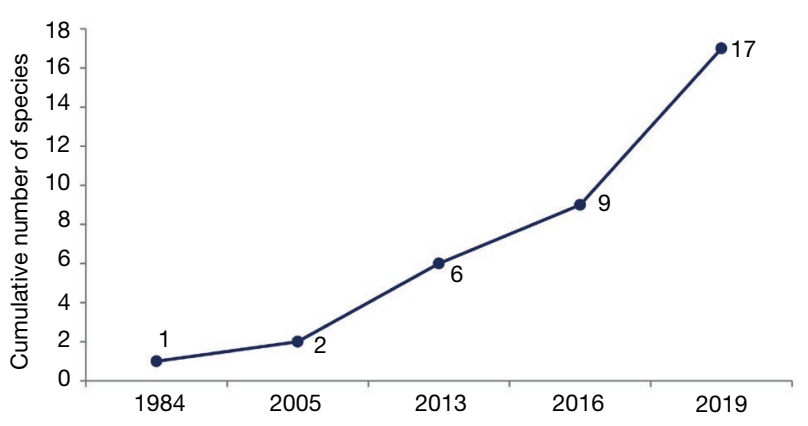

FIG. 16. - The cumulative number of discovered species of Dryinidae Haliday, 1833 from Iran (1984 to 2019).

Stål, 1866. Dryinus species are parasitoids of Fulgoromorpha Latreille, 1807, and their hosts in the Western Palaearctic subregion are known only for D. tarraconensis (Guglielmino \& Olmi 2007; Guglielmino et al. 2013).

An overall analysis of the discovered Dryinidae in Iran (Fig. 16) reveals that very little information was added until 2005, and also the lack of focus on this important group of biological control agents. The steep rise of the curve between 2005-2019 reveals the importance of efficient samplings, supplemented by the recent findings in Derafshan et al. (2016, 2017,2020 ), with a significant number of the newly recorded species. According to the sporadical samplings, many areas particularly in the rainforest of the north, mountain forests in the west and semi-desertic parts in the south of the country remained unexplored. So that many other species of Dryinidae (including endemic species) are yet to be discovered by the subsequent studies.

\section{Conflict of interests}

The authors declare that they have no known competing financial interests or personal relationships that could have appeared to influence the work reported in this paper.

\section{Acknowledgements}

First of all, we thank our team members in the Department of Plant Protection, University of Zabol, for their assistance during the sampling, which enhanced this contribution. Funding for this study was supported by the University of Zabol (No. UOZ-GR-9618-6) to ER. Many thanks to Prof. Michael von Tschirnhaus (Bielefeld, Germany) for sending on loan specimens of Aphelopus orphanidesi collected in Germany. The thourough comments and suggestions by the expert reviewers, Dr Robert S. Copeland and Dr Arkady S. Lelej, greatly improved the quality of the manuscript.

\section{REFERENCES}

Ashmead W. H. 1893. - Monograph of the North American Proctotrypidae. Bulletin of the United States National Museum 45: 1-472. https://doi.org/10.5479/si.03629236.45.1

Azevedo C. O., Alencar I. D. C. C., Ramos M. S., Barbosa D. N., Colombo W. D., Vargas J. M. \& Lim J. 2018. — Global guide to the flat wasps (Hymenoptera, Bethylidae). Zootaxa 4489: 1-294. https://doi.org/10.11646/zootaxa.4489.1.1

DALMAN J. W. 1818. - Nagra nya genera och species af insekter beskrifna. Kongliga Svenska Vetenskaps-Akademiens Handlingar 39: 69-89.

Dalman J. W. 1823. - Analecta entomologica. Typis Lindhianis, Holmiae, 104 p. https://doi.org/10.5962/bhl.title.66069

DeRAFSHAN H. A., RAKHSHANi E. \& Olmi M. 2016. — A review of 
the genus Dryinus Latreille, 1804 (Hymenoptera, Chrysidoidea, Dryinidae) from Iran, with description of a new species. Zootaxa 4117: 411-420. https://doi.org/10.11646/zootaxa.4117.3.8

Derafshan H. A., RaKHSHANi E. \& Olmi M. 2017. - Discovery of the genus Mirodryinus Ponomarenko, 1972 (Hymenoptera, Chrysidoidea, Dryinidae) associated with salt cedar trees in Eastern part of Iran. Turkish Journal of Zoology 41: 345-353. https:// doi.org/10.3906/zoo-1605-35

Ghafouri Moghaddam M., Rakhshani E., Arabzadeh MA., Derafshan Ha. \& KaVAllieratos NG. 2019. - The Stephanidae (Hymenoptera, Stephanoidea) of Iran with the description of a new species. Insect Systematics \& Evolution 50: 583-600. https:// doi.org/10.1163/1876312X-00002191

Derafshan H. A., Olmi M., Ghafouri Moghaddam M. \& RAKHSHANI E. 2020. - Taxonomic study of the subfamily Gonatopodinae Kieffer (Hymenoptera, Chrysidoidea, Dryinidae) from Iran with the description of a new species. Zootaxa 4789: 371-402. https://doi.org/10.11646/zootaxa.4789.2.3

Guglielmino A. \& Olmi M. 2007. - A host-parasite catalog of world Dryinidae (Hymenoptera: Chrysidoidea): second supplement. Bollettino di Zoologia agraria e Bachicoltura, Series II 39 (2): 121-129.

Guglielmino A., Olmi M. \& BÜCKLe C. 2013. — An updated host-parasite catalogue of world Dryinidae (Hymenoptera: Chrysidoidea). Zootaxa 3740 (1): 1-113. https://doi.org/10.11646/ zootaxa.3740.1.1

Guglielmino A., Olmi M., LiU J-X. \& Contarini M. 2019. — An updated checklist of the extant Western Palaearctic Dryininae (Hymenoptera, Dryinidae). ZooKeys 874: 83-99. https://doi. org/10.3897/zookeys.874.33226

HALIDAY A. H. 1833. - An essay on the classification of the Parasitic Hymenoptera of Britain, which correspond with the Ichneumones minuti of Linnaeus. The Entomological Magazine 1: 259-273.

Heraty J. M., Derafshan H. A. \& Ghafouri Moghaddam M. 2019. - Review of the Philomedinae Ruschka (Hymenoptera: Chalcidoidea: Perilampidae), with description of three new species. Arthropod Systematics \& Phylogeny 77: 39-56. https://doi. org/10.26049/ASP77-1-2019-03

JURINE L. 1807. — Nouvelle méthode de classer les Hyménoptères et les Diptères, 1. Hyménoptères. Paschoud, Genève, 319 p. [Switzerland]

KaWAda R, Lanes G. O. \& Azevedo C. O. 2015. - Evolution of metapostnotum in flat wasps (Hymenoptera, Bethylidae): implications for homology assessments in Chrysidoidea. Plos One 10 (10): e0140051. https://doi.org/10.1371/journal.pone.0140051

KiefFer J. J. \& Marshall T. A. 1906. - Proctotrypidae. In André E. (Ed.), Species des Hyménoptères d'Europe et d'Algerie. Vol. 9. Hermann, Paris: 289-552.

LATREILle P. A. 1804. — Tableau méthodique des insectes. In: Société de Naturalistes et d'Agriculteurs (ed.), Nouvelle dictionnaire d'Histoire naturelle. Vol. 24. Déterville, Paris: 129-200.

LATREILlE P. A. 1805. - Histoire naturelle generale et particulière des crustacés et des insectes. F. Dufart, Paris, 13: 432 p.

LJUNGH S. J. 1810. — Gonatopus, novum insectorum genus. Beiträge zur Naturkunde 2: 161-163

Marshall T. A. 1868. - On some British Diapriidae. The Entomologist's Monthly Magazine 4: 201-209.

Olmi M. \& OrPhANides G. M. 1994. - A new species of the genus Aphelopus from Cyprus (Hymenoptera Drynidae). Bollettino del Museo Regionale di Scienze Naturali di Torino 12 (2): 407-412.

Olmi M. \& Van Harten A. 2000. — Notes on Dryinidae, Embolemi- dae and Sclerogibbidae (Hymenoptera: Chrysidoidea) of Yemen, with keys to the species of the Arabian Peninsula. Fauna of Arabia 18: 253-271.

Olmi M. \& Virla E. 2014. - Dryinidae of the Neotropical region (Hymenoptera: Chrysidoidea).Zootaxa, 792 (1): 001-534. https:// doi.org/10.11646/zootaxa.3792.2.1

Olmi M. \& Xu Z. 2015. - Dryinidae of the Eastern Palaearctic region (Hymenoptera: Chrysidoidea). Zootaxa, 3996 (1): 1-253. https://doi.org/10.11646/zootaxa.3996.1.1

Olmi M. 1984. - A revision of the Dryinidae (Hymenoptera). Memoirs of the American Entomological Institute 37: I-XII + 1-1913

Olmi M. 1987. - Descrizione di nuove specie di Dryinidae (Hymenoptera Chrysidoidea). Bollettino di Zoologia agraria e di Bachicoltura, Ser. II, 19: 31-70.

Olmi M. 1994. - The Dryinidae and Embolemidae (Hymenoptera: Chrysidoidea) of Fennoscandia and Denmark. Fauna Entomologica Scandinavica 30: 1-100. [E. J. Brill, Leiden, Netherlands]

Olmi M. 1998 [1997]. — New Embolemidae and Dryinidae (Hymenoptera Chrysidoidea). Frustula entomologica, New Series 20 (33): 30-118

Olmi M. 2005. - Dryinus gharaeii sp. nov. from Iran (Hymenoptera: Chrysidoidea, Dryinidae). Entomologist's Gazette 56: 207-209.

Olmi M. 2006 ["2005"]. - A catalogue of Dryinidae and Embolemidae of South Africa, with descriptions of new species (Hymenoptera Chrysidoidea). Frustula entomologica (N.S.) 28-29 (41-42): 1-57.

Olmi M. 2020. — Biodiversity of the aculeate wasps (Hymenoptera: Aculeata) of the Arabian Peninsula: Chrysidoidea, Dryinidae. Zootaxa 4754 (1): 122-128. https://doi.org/10.11646/ zootaxa.4754.1.11

Olmi M., Copeland R. S. \& VAN NoORT S. 2019. — Dryinidae of the Afrotropical region (Hymenoptera, Chrysidoidea). Zootaxa, 4630 (1): 1-619. https://doi.org/10.11646/zootaxa.4630.1.1

Olmi M., Virla E. \& FernándeZ F. 2000. — "Las avispas Dryinidae de la región Neotropical (Hymenoptera: Chrysidoidea)." Biota Colombiana 1 (2): 141-163.

PERKINS R. C. L. 1903. - The leafhopper of the sugar cane. Territory of Hawaii, Board of Agriculture and Forest, Division of Entomology, Bulletin 1: 1-38.

PERKINS R. C. L. 1905. - Leafhoppers and their natural enemies (Pt. I. Dryinidae). Report of Work of the Experiment Station of the Hawaiian Sugar Planters' Association, Division of Entomology, Bulletin 1 (1): 1-69.

PerKINS R. C. L. 1912. — Parasites of the Family Dryinidae. Report of Work of the Experiment Station of the Hawaiian Sugar Planters' Association, Division of Entomology, Bulletin 11: 5-20.

PonOMARENKO N. G. 1972. - [On the fauna of dryinids (Hymenoptera, Dryinidae) of the Mongolian People's Republic]. [Insects of Mongolia] 1: 673-680. (In Russian).

RicHARDS O. W. 1939. - The British Bethylidae (s.l.) (Hymenoptera). The Transactions of the Royal entomological Society of London 89: 185-344. https://doi.org/10.1111/j.1365-2311.1939.tb00740.x

ShorThouse D. P. 2010. - SimpleMappr, an online tool to produce publication-quality point maps. http://www.simplemappr. net [Accessed October 26, 2019]

VAn ACHTERBerg C. 2009. - Can Townes type Malaise traps be improved? Some recent developments. Entomologische Berichten 69: 129-135.

WestwOOD J. O. 1840. - An Introduction to the Modern Classification of Insects. Vol. 2. Longman, Orme, Brown, Green and Longmans, London, 587 p. 